\title{
High-Speed Transmission in Long-Haul Electrical Systems
}

\author{
Beatriz Juárez-Campos, ${ }^{1}$ Elena I. Kaikina $(\mathbb{D})^{2}$ \\ Pavel I. Naumkin $(\mathbb{D})^{2}$ and Héctor Francisco Ruiz-Paredes ${ }^{1}$ \\ ${ }^{1}$ Instituto Tecnológico de Morelia, Avenida Tecnológico No. 1500, Lomas de Santiaguito, 58120 Morelia, MICH, Mexico \\ ${ }^{2}$ Centro de Ciencias Matemáticas, UNAM Campus Morelia, AP 61-3 Xangari, 58089 Morelia, MICH, Mexico \\ Correspondence should be addressed to Elena I. Kaikina; ekaikina@matmor.unam.mx
}

Received 29 January 2018; Accepted 12 March 2018; Published 18 April 2018

Academic Editor: Sining Zheng

Copyright (C) 2018 Beatriz Juárez-Campos et al. This is an open access article distributed under the Creative Commons Attribution License, which permits unrestricted use, distribution, and reproduction in any medium, provided the original work is properly cited.

We study the equations governing the high-speed transmission in long-haul electrical systems $i \partial_{t} u-(1 / 3)\left|\partial_{x}\right|^{3} u=i \lambda \partial_{x}\left(|u|^{2} u\right)$, $(t, x) \in \mathbb{R}^{+} \times \mathbb{R}, u(0, x)=u_{0}(x), x \in \mathbb{R}$, where $\lambda \in \mathbb{R},\left|\partial_{x}\right|^{\alpha}=\mathscr{F}^{-1}|\xi|^{\alpha} \mathscr{F}$, and $\mathscr{F}$ is the Fourier transformation. Our purpose in this paper is to obtain the large time asymptotics for the solutions under the nonzero mass condition $\int u_{0}(x) d x \neq 0$.

\section{Introduction}

We study the equations governing the high-speed transmission in long-haul electrical systems

$$
\begin{aligned}
i \partial_{t} u-\frac{1}{3}\left|\partial_{x}\right|^{3} u & =i \lambda \partial_{x}\left(|u|^{2} u\right), \quad(t, x) \in \mathbb{R}^{+} \times \mathbb{R}, \\
u(0, x) & =u_{0}(x), \quad x \in \mathbb{R},
\end{aligned}
$$

where $\lambda \in \mathbb{R},\left|\partial_{x}\right|^{3}=\mathscr{F}^{-1}|\xi|^{3} \mathscr{F}$, and $\mathscr{F}$ is the Fourier transformation defined by $\mathscr{F} \phi=(1 / \sqrt{2 \pi}) \int_{\mathbb{R}} e^{-i x \xi} \phi d x$. Note that we have the relation $u(-t, x)=\bar{u}(t,-x)$, so we can only consider the case $t>0$. For the regular solution of (1) we have the conservation law $\|u(t)\|_{\mathbf{L}^{2}}=\left\|u_{0}\right\|_{\mathbf{L}^{2}}$. We are interested in the case of nonzero mass condition $\int_{\mathbb{R}} u_{0}(x) d x \neq 0$. By (1) we get the conservation of the mass $\int_{\mathbb{R}} u(t, x) d x=\int_{\mathbb{R}} u_{0}(x) d x \neq$ 0 for all $t>0$.

This equation arises in the context of high-speed soliton transmission in long-haul optical communication system [1]. Also it can be considered as a particular form of the higher order nonlinear Schrödinger equation introduced by [2] to describe the nonlinear propagation of pulses through optical fibers. This equation also represents the propagation of pulses by taking higher dispersion effects into account than those given by the Schrödinger equation (see [3-11]).
The higher order nonlinear Schrödinger equations have been widely studied recently. For the local and global wellposedness of the Cauchy problem we refer to [12-14] and references cited therein. The dispersive blow-up was obtained in [15]. The existence and uniqueness of solutions to (1) were proved in [16-25] and the smoothing properties of solutions were studied in [18-21, 24, 26-31]. The blow-up effect for a special class of slowly decaying solutions of Cauchy problem (1) was found in [32].

As far as we know the question of the large time asymptotics for solutions to Cauchy problem (1) is an open problem. We develop here the factorization technique originated in our previous papers [33-38].

We denote the Lebesgue space by $\mathbf{L}^{p}=\left\{\phi \in \mathbf{S}^{\prime} ;\|\phi\|_{\mathbf{L}^{p}}<\right.$ $\infty\}$, where the norm $\|\phi\|_{L^{p}}=\left(\int|\phi(x)|^{p} d x\right)^{1 / p}$ for $1 \leq p<$ $\infty$ and $\|\phi\|_{L^{\infty}}=\sup _{x \in \mathbb{R}}|\phi(x)|$. The weighted Sobolev space is $\mathbf{H}_{p}^{m, s}=\left\{\varphi \in \mathbf{S}^{\prime} ;\|\phi\|_{\mathbf{H}_{p}^{m, s}}=\left\|\langle x\rangle^{s}\left\langle i \partial_{x}\right\rangle^{m} \phi\right\|_{\mathbf{L}^{p}}<\infty\right\}$, where $m, s \in \mathbb{R}, 1 \leq p \leq \infty,\langle x\rangle=\sqrt{1+x^{2}}$, and $\left\langle i \partial_{x}\right\rangle=\sqrt{1-\partial_{x}^{2}}$. We also use the notations $\mathbf{H}^{m, s}=\mathbf{H}_{2}^{m, s}$, $\mathbf{H}^{m}=\mathbf{H}^{m, 0}$ shortly, if it does not cause any confusion. Let $\mathbf{C}(\mathbf{I} ; \mathbf{B})$ be the space of continuous functions from an interval I to a Banach space B. Different positive constants might be denoted by the same letter $C$. We denote by $\mathscr{F} \phi$ or $\widehat{\phi}(\xi)=(1 / \sqrt{2 \pi}) \int_{\mathbb{R}} e^{-i x \xi} \phi(x) d x$ the Fourier transform of the 
function $\phi$, then the inverse Fourier transformation is given by $\mathscr{F}^{-1} \phi=(1 / \sqrt{2 \pi}) \int_{\mathbb{R}} e^{i x \xi} \phi(\xi) d \xi$.

We are now in a position to state our result.

Theorem 1. Assume that the initial data $u_{0} \in \mathbf{H}^{1} \cap \mathbf{H}^{0,1}$ have a sufficiently small norm $\left\|u_{0}\right\|_{\mathbf{H}^{1} \cap \mathrm{H}^{0,1}} \leq \varepsilon$. Then there exists a unique global solution $\mathscr{F} e^{-(i t / 3)\left|\partial_{x}\right|^{3}} u \in \mathbf{C}\left([0, \infty) ; \mathbf{L}^{\infty} \cap \mathbf{H}^{0,1}\right)$ of Cauchy problem (1). Furthermore the estimate

$$
\begin{aligned}
& \sup _{t>0}\left(\left\|\mathscr{F} e^{-(i t / 3)\left|\partial_{x}\right|^{3}} u(t)\right\|_{\mathbf{L}^{\infty}}\right. \\
& +\langle t\rangle^{-1 / 6}\left\|x e^{-(i t / 3)\left|\partial_{x}\right|^{3}} u(t)\right\|_{\mathbf{L}^{2}} \\
& \left.+\langle t\rangle^{(1 / 3)(1-1 / p)}\|u(t)\|_{\mathbf{L}^{p}}\right) \leq C \varepsilon
\end{aligned}
$$

is true, where $p>4$.

Next we prove the existence of the self-similar solutions $v_{m}(t, x)=t^{-1 / 3} f_{m}\left(x t^{-1 / 3}\right)$.

Theorem 2. There exists a unique solution of Cauchy problem (1) in the self-similar form $v_{m}(t, x)=t^{-1 / 3} f_{m}\left(x t^{-1 / 3}\right)$, such that

$$
m=\frac{1}{\sqrt{2 \pi}} \int_{\mathbf{R}} v_{m}(t, x) d x=\frac{1}{\sqrt{2 \pi}} \int_{\mathbf{R}} f_{m}(x) d x \neq 0,
$$

where $m$ is sufficiently small number and

$$
\begin{gathered}
\mathscr{F} e^{-(i t / 3)\left|\partial_{x}\right|^{3}} v_{m} \in \mathbf{C}\left([1, \infty) ; \mathbf{L}^{\infty}\right), \\
x e^{-(i t / 3)\left|\partial_{x}\right|^{3}} v_{m} \in \mathbf{C}\left([1, \infty) ; \mathbf{L}^{2}\right) .
\end{gathered}
$$

Furthermore the estimate

$$
\begin{aligned}
& \sup _{t>1}\left(\left\|\mathscr{F} e^{-(i t / 3)\left|\partial_{x}\right|^{3}} v_{m}(t)\right\|_{\mathbf{L}^{\infty}}\right. \\
& +t^{-1 / 6}\left\|x e^{-(i t / 3)\left|\partial_{x}\right|^{3}} v_{m}(t)\right\|_{\mathbf{L}^{2}} \\
& \left.+t^{(1 / 3)(1-1 / p)}\left\|v_{m}(t)\right\|_{\mathbf{L}^{p}}\right) \leq C|m|
\end{aligned}
$$

is true, where $p>4$.

Now we state the stability of solutions to Cauchy problem (1) in the neighborhood of the self-similar solution $v_{m}(t, x)$.

Theorem 3. Suppose that

$$
\frac{1}{\sqrt{2 \pi}} \int_{\mathbf{R}} f_{m}(x) d x=\frac{1}{\sqrt{2 \pi}} \int_{\mathbf{R}} u_{0}(x) d x=m \neq 0 .
$$

Let $u(t, x)$ and $v_{m}(t, x)$ be the solutions constructed in Theorems 1 and 2, respectively. Then there exists small $\gamma>0$ such that the asymptotics

$$
\left\|u(t)-v_{m}(t)\right\|_{L^{\infty}} \leq C t^{-1 / 2+\gamma}
$$

are true for $t \geq 1$.
Our approach is based on the factorization techniques. Define the free evolution group $\mathscr{U}(t)=\mathscr{F}^{-1} e^{-(i t / 3)|\xi|^{3}} \mathscr{F}$ and write

$$
\mathcal{U}(t) \mathscr{F}^{-1} \phi=\mathscr{D}_{t} \sqrt{\frac{|t|}{2 \pi}} \int_{\mathbb{R}} e^{i t\left(x \xi-(1 / 3)|\xi|^{3}\right)} \phi(\xi) d \xi,
$$

where $\mathscr{D}_{t} \phi=|t|^{-1 / 2} \phi(x / t)$ is the dilation operator. There is a unique stationary point $\xi=\mu(x) \equiv(x /|x|) \sqrt{|x|}$ in the integral $\int_{\mathbb{R}} e^{i t\left(x \xi-(1 / 3)|\xi|^{3}\right)} \phi(\xi) d \xi$, which is defined as the root of the equation $\xi|\xi|=x$ for all $x \in \mathbb{R}$. Define the scaling operator $(\mathscr{B} \phi)(x)=\phi(\mu(x))$. Hence we find the following decomposition $\mathcal{U}(t) \mathscr{F}^{-1} \phi=\mathscr{D}_{t} \mathscr{B} M \mathscr{V} \phi$, where the multiplication factor $M=e^{(2 i t / 3)|\eta|^{3}}$ and the deformation operator

$$
\mathscr{V}(t) \phi=\sqrt{\frac{|t|}{2 \pi}} \int_{\mathbb{R}} e^{-i t S(\xi, \eta)} \phi(\xi) d \xi
$$

where the phase function $S(\xi, \eta)=(1 / 3)|\xi|^{3}-(1 / 3)|\eta|^{3}-$ $\eta|\eta|(\xi-\eta)$. Denote $\mathscr{A}_{k}=\bar{M}^{k}(1 / 2 t|\eta|) \partial_{\eta} M^{k}, k=0,1$. We have $\mathscr{A}_{1}=\mathscr{A}_{0}+i \eta$, and also $\mathscr{A}_{1} \mathscr{V}=\mathscr{V} i \xi$, $[i \eta, \mathscr{V}]=-\mathscr{A}_{0} \mathscr{V}$; therefore we obtain the commutator $\partial_{\eta} \mathscr{V}=-2 t|\eta|[i \eta, \mathscr{V}]$. Since $\partial_{\xi} S(\xi, \eta)=\xi|\xi|-\eta|\eta|$, then we get $i t[\eta|\eta|, \mathscr{V}] \phi=$ $-\mathscr{V} \partial_{\xi} \phi$. Also we need the representation for the inverse evolution group $\mathscr{F} \mathscr{U}(-t) \phi=\mathscr{V}^{*} \bar{M} \mathscr{B}^{-1} \mathscr{D}_{t}^{-1}$, where the inverse dilation operator $\mathscr{D}_{t}^{-1} \phi=|t|^{1 / 2} \phi(x t)$, the inverse scaling operator $\left(\mathscr{B}^{-1} \phi\right)(\eta)=\phi(\eta|\eta|)$, and the inverse deformation operator

$$
\mathscr{V}^{*}(t) \phi=\sqrt{\frac{2|t|}{\pi}} \int_{\mathbb{R}} e^{i t S(\xi, \eta)} \phi(\eta)|\eta| d \eta .
$$

We have $i \xi \mathscr{V}^{*} \phi=\mathscr{V}^{*} \mathscr{A}_{1} \phi$. Hence the commutator $\left[i \xi, \mathscr{V}^{*}\right]=\mathscr{V}^{*} \mathscr{A}_{0}$. Define the new dependent variable $\widehat{\varphi}=$ $\mathscr{F} \mathscr{U}(-t) u(t)$. Since $\mathscr{F} \mathscr{U}(-t) \mathscr{L}=\partial_{t} \mathscr{F} \mathscr{U}(-t)$ with $\mathscr{L}=\partial_{t}+$ $(i / 3)\left|\partial_{x}\right|^{3}$, applying the operator $\mathscr{F} \mathscr{U}(-t)$ to (1), substituting $u(t)=\mathscr{U}(t) \mathscr{F}^{-1} \widehat{\varphi}=\mathscr{D}_{t} \mathscr{B} M \mathscr{V} \widehat{\varphi}$, and using the factorization techniques, we get

$$
\begin{aligned}
\partial_{t} \widehat{\varphi} & =\mathscr{F} \mathscr{U}(-t) \mathscr{L} u=i \lambda \xi \mathscr{F} \mathscr{U}(-t)\left(|u|^{2} u\right) \\
& =i \lambda \xi \mathscr{V}^{*} \bar{M} \mathscr{B}^{-1} \mathscr{D}_{t}^{-1}\left(\left|\mathscr{D}_{t} \mathscr{B} M \mathscr{V} \hat{\varphi}\right|^{2} \mathscr{D} \mathscr{B} M \mathscr{V} \hat{\varphi}\right) \\
& =i \lambda \xi t^{-1} \mathscr{V}^{*} \bar{M} \mathscr{B}^{-1}\left(|\mathscr{B} M \mathscr{V} \hat{\varphi}|^{2} \mathscr{B} M \mathscr{V} \hat{\varphi}\right) \\
& =i \lambda \xi t^{-1} \mathscr{V}^{*} \bar{M}\left(|M \mathscr{V} \hat{\varphi}|^{2} M \mathscr{V} \hat{\varphi}\right) \\
& =i \lambda \xi t^{-1} \mathscr{V}^{*}\left(|\mathscr{V} \phi|^{2} \mathscr{V} \phi\right)
\end{aligned}
$$

since the nonlinearity is gauge invariant. Finally we mention some important identities. The operator $\mathcal{J}=\mathscr{U}(t) x \mathcal{U}(-t)=$ $x+i t \partial_{x}\left|\partial_{x}\right|$ plays a crucial role in the large time asymptotic estimates. Note that $\mathscr{J}$ commutes with $\mathscr{L}$, that is, $[\mathscr{J}, \mathscr{L}]=0$. To avoid the derivative loss we also use the operator $\mathscr{P}=$ $3 t \partial_{t}+\partial_{x} x$. Note the commutator relation $\left[\widehat{\mathscr{P}}, e^{-(i t / 3)|\xi|^{3}}\right]=0$ 
with $\widehat{\mathscr{P}}=3 t \partial_{t}-\xi \partial_{\xi}$. Thus using $u(t)=\mathscr{U}(t) \mathscr{F}^{-1} \widehat{\varphi}=$ $\mathscr{D}_{t} M \mathscr{V} \hat{\varphi}=\mathscr{F}^{-1} e^{-(i t / 3)|\xi|^{3}} \hat{\varphi}$, we get $\mathscr{P} u=\mathscr{U}(t) \mathscr{F}^{-1} \widehat{\mathscr{P}} \hat{\varphi}$. Also we have the identity $\mathscr{J}=\partial_{x}^{-1} \mathscr{P}-3 t \partial_{x}^{-1} \mathscr{L}$ and $[\mathscr{L}, \mathscr{P}]=3 \mathscr{L}$ holds.

\section{Estimates in the Uniform Norm}

2.1. Kernels. Define the kernel

$$
A_{j}(t, \eta)=\sqrt{\frac{|t|}{2 \pi}} \int_{\mathbb{R}} e^{-i t s(\xi, \eta)} \Theta\left(\xi \eta^{-1}\right) \xi^{j} d \xi
$$

for $\eta \neq 0$, where the cutoff function $\Theta(z) \in \mathbf{C}^{2}(\mathbb{R})$ is such that $\Theta(z)=0$ for $z \leq 1 / 3$ or $z \geq 3$ and $\Theta(z)=$ 1 for $2 / 3 \leq z \leq 3 / 2$. We change $\xi=\eta y$, then we get $A_{j}(t, \eta)=|\eta| \eta^{j} \sqrt{|t| / 2 \pi} \int_{1 / 3}^{3} e^{-i t|\eta|^{3} G(y)} \Theta(y) y^{j} d y$, where $S(\eta y, \eta)=|\eta|^{3} G(y)$ and $G(y)=(1 / 3)(y+2)(y-1)^{2}, y>0$. To compute the asymptotics of the kernel $A_{j}(t, \eta)$ for large $t$ we apply the stationary phase method (see [39], p. 110)

$$
\begin{aligned}
& \int_{\mathbb{R}} e^{i z g(y)} f(y) d y \\
& =e^{i z g\left(y_{0}\right)} f\left(y_{0}\right) \sqrt{\frac{2 \pi}{z\left|g^{\prime \prime}\left(y_{0}\right)\right|} e^{i(\pi / 4) \operatorname{sgn} g^{\prime \prime}\left(y_{0}\right)}} \\
& \quad+O\left(z^{-3 / 2}\right)
\end{aligned}
$$

for $z \rightarrow+\infty$, where the stationary point $y_{0}$ is defined by the equation $g^{\prime}\left(y_{0}\right)=0$. By virtue of formula (13) with $g(y)=$ $-G(y), f(y)=\Theta(y) y^{j}$, and $y_{0}=1$, we get

$$
A_{j}(t, \eta)=\frac{t^{1 / 2}|\eta| \eta^{j}}{\sqrt{2 i\left\langle t \eta^{3}\right\rangle}}+O\left(t^{1 / 2} \eta^{1+j}\left\langle t \eta^{3}\right\rangle^{-1}\right)
$$

for $t \eta^{3} \rightarrow \infty$. Also we have the estimate $\left|A_{j}(t, \eta)\right| \leq$ $C t^{1 / 2}|\eta|^{j+1}\left\langle t \eta^{3}\right\rangle^{-1 / 2}$.

In the same manner changing $\eta=\xi y$, we get for the kernel

$$
\begin{aligned}
A^{*}(t, \xi) & =\sqrt{\frac{2|t|}{\pi}} \int_{\mathbb{R}} e^{i t S(\xi, \eta)} \Theta\left(\eta \xi^{-1}\right)|\eta| d \eta \\
& =\xi^{2} \sqrt{\frac{2|t|}{\pi}} \int_{1 / 3}^{3} e^{i t|\xi|^{3} \widetilde{G}(y)} \Theta(y)|y| d y
\end{aligned}
$$

for $\xi \neq 0$, where $S(\xi, \xi y)=|\xi|^{3} \widetilde{G}(y)$ with $\widetilde{G}(y)=(1 / 3)(2 y+$ 1) $(y-1)^{2}, y>0$. Then by virtue of formula (13) with $g(y)=$ $\widetilde{G}(y), f(y)=\Theta(y)|y|$, and $y_{0}=1$, we obtain

$$
A^{*}(t, \xi)=2 t^{1 / 2} \xi^{2} \sqrt{\frac{2 i}{\left\langle t \xi^{3}\right\rangle}}+O\left(t^{1 / 2} \xi^{2}\left\langle t \xi^{3}\right\rangle^{-1}\right)
$$

for $t \xi^{3} \rightarrow \infty$. Also we have the estimate $\left|A^{*}(t, \xi)\right| \leq$ $C t^{1 / 2} \xi^{2}\left\langle t \xi^{3}\right\rangle^{-1 / 2}$.
2.2. Asymptotics for the Operator $\mathscr{V}$. In the next lemma we estimate the operator $\mathscr{V}$ in the uniform norm. Define the cutoff function $\chi_{1}(z) \in \mathbf{C}^{2}(\mathbb{R})$ such that $\chi_{1}(z)=0$ for $|z| \geq 3$ and $\chi_{1}(z)=1$ for $|z| \leq 2$ and $\chi_{2}(z)=1-\chi_{1}(z)$. Consider two operators

$$
\mathscr{V}_{j}(t) \phi=\sqrt{\frac{|t|}{2 \pi}} \int_{\mathbb{R}} e^{-i t S(\xi, \eta)} \phi(\xi) \chi_{j}\left(\xi \eta^{-1}\right) d \xi,
$$

so that we have $\mathscr{V}(t) \phi=\mathscr{V}_{1}(t) \phi+\mathscr{V}_{2}(t) \phi$ for $\eta \neq 0$. Define the norm $\|\phi\|_{\mathbf{Y}}=\|\phi\|_{\mathbf{L}^{\infty}}+t^{-1 / 6}\left\|\partial_{\xi} \phi\right\|_{\mathbf{L}^{2}}$.

Lemma 4. The following estimates $\left|\mathscr{V}_{1} \xi^{j} \phi-A_{j}(t, \eta) \phi(\eta)\right| \leq$ $C t^{1 / 2}|\eta|^{j+1}\left\langle t^{1 / 3} \eta\right\rangle^{-7 / 4}\|\phi\|_{\mathbf{Y}}$ if $j \geq 0$ and $\left|\mathscr{V}_{2} \xi^{j} \phi\right| \leq$ $C t^{1 / 6-j / 3}\left\langle t^{1 / 3} \eta\right\rangle^{j-3 / 2}\|\phi\|_{\mathbf{Y}}$ if $j=0,1$ are valid for all $t \geq 1$, $\eta \neq 0$.

Proof. We write

$$
\begin{gathered}
\mathscr{V}_{1} \xi^{j} \phi-A_{j} \phi=\sqrt{\frac{|t|}{2 \pi}} \int_{\mathbb{R}} e^{-i t S(\xi, \eta)}(\phi(\xi)-\phi(\eta)) \\
\cdot \Theta\left(\xi \eta^{-1}\right) \xi^{j} d \xi+\sqrt{\frac{|t|}{2 \pi}} \int_{\mathbb{R}} e^{-i t S(\xi, \eta)} \phi(\xi) \\
\cdot\left(\chi_{1}\left(\xi \eta^{-1}\right)-\Theta\left(\xi \eta^{-1}\right)\right) \xi^{j} d \xi=I_{1}+I_{2}
\end{gathered}
$$

for $\eta \neq 0$. For the summand $I_{1}$ we integrate by parts via identity

$$
e^{-i t S(\xi, \eta)}=H_{1} \partial_{\xi}\left((\xi-\eta) e^{-i t S(\xi, \eta)}\right)
$$

with $H_{1}=\left(1-i t(\xi-\eta) \partial_{\xi} S(\xi, \eta)\right)^{-1}$, to get

$$
\begin{aligned}
I_{1} & =C t^{1 / 2} \int_{\mathbb{R}} e^{-i t S(\xi, \eta)}(\phi(\xi)-\phi(\eta))(\xi-\eta) \\
& \cdot \partial_{\xi}\left(H_{1} \Theta\left(\xi \eta^{-1}\right) \xi^{j}\right) d \xi \\
& +C t^{1 / 2} \int_{\mathbb{R}} e^{-i t S(\xi, \eta)}(\xi-\eta) H_{1} \Theta\left(\xi \eta^{-1}\right) \\
& \cdot \xi^{j} \partial_{\xi} \phi(\xi) d \xi .
\end{aligned}
$$

We find the estimates

$$
\begin{aligned}
& \left|H_{1} \Theta\left(\xi \eta^{-1}\right) \xi^{j}\right|+\left|(\xi-\eta) \partial_{\xi}\left(H_{1} \Theta\left(\xi \eta^{-1}\right) \xi^{j}\right)\right| \\
& \quad \leq \frac{C|\eta|^{j}}{1+t|\eta|(\xi-\eta)^{2}}
\end{aligned}
$$

in the domain $1 / 3<\xi / \eta<3$. Therefore we obtain

$$
\begin{aligned}
\left|I_{1}\right| \leq C t^{1 / 2}|\eta|^{j} & \\
& \cdot \int_{1 / 3<\xi / \eta<3} \frac{|\phi(\xi)-\phi(\eta)|}{|\xi-\eta|} \frac{|\xi-\eta| d \xi}{1+t|\eta|(\xi-\eta)^{2}} \\
& +C t^{1 / 2}|\eta|^{j} \int_{1 / 3<\xi / \eta<3} \frac{|\xi-\eta|\left|\partial_{\xi} \phi(\xi)\right| d \xi}{1+t|\eta|(\xi-\eta)^{2}} .
\end{aligned}
$$


By the Hardy inequality $\int_{1 / 3<\xi / \eta<3}\left(|\phi(\xi)-\phi(\eta)|^{2} /|\xi-\eta|^{2}\right) d \xi \leq$ $C\left\|\partial_{\xi} \phi\right\|_{\mathrm{L}^{2}}^{2}$ and by the Cauchy-Schwarz inequality, changing $\xi=\eta y$ we find

$$
\begin{aligned}
&\left|I_{1}\right| \leq C t^{1 / 2}|\eta|^{j}\left\|\partial_{\xi} \phi\right\|_{\mathbf{L}^{2}} \\
& \cdot\left(\int_{1 / 3<\xi / \eta<3} \frac{(\xi-\eta)^{2} d \xi}{\left(1+t|\eta|(\xi-\eta)^{2}\right)^{2}}\right)^{1 / 2} \\
& \quad \leq C t^{1 / 2}|\eta|^{j+3 / 2}\left\|\partial_{\xi} \phi\right\|_{\mathbf{L}^{2}} \\
& \cdot\left(\int_{1 / 3}^{3} \frac{(y-1)^{2} d y}{\left(1+|t||\eta|^{3}(y-1)^{2}\right)^{2}}\right)^{1 / 2} \\
& \quad \leq C t^{1 / 2}\left\|\partial_{\xi} \phi\right\|_{L^{2}}|\eta|^{j+3 / 2}\left\langle t \eta^{3}\right\rangle^{-3 / 4} .
\end{aligned}
$$

To estimate the integral $I_{2}$ we integrate by parts via the identity

$$
e^{-i t S(\xi, \eta)}=H_{2} \partial_{\xi}\left(\xi e^{-i t S(\xi, \eta)}\right)
$$

with $H_{2}=\left(1-i t \xi \partial_{\xi} S(\xi, \eta)\right)^{-1}$, to get

$$
\begin{aligned}
I_{2}= & C t^{1 / 2} \phi(0) \\
& \cdot \int_{\mathbb{R}} e^{-i t S(\xi, \eta)} \xi \partial_{\xi}\left(\left(\chi_{1}\left(\xi \eta^{-1}\right)-\Theta\left(\xi \eta^{-1}\right)\right) H_{2} \xi^{j}\right) d \xi \\
& +C t^{1 / 2} \int_{\mathbb{R}} e^{-i t S(\xi, \eta)} \\
& \cdot \frac{\phi(\xi)-\phi(0)}{\xi} \xi^{2} \partial_{\xi}\left(\left(\chi_{1}\left(\xi \eta^{-1}\right)-\Theta\left(\xi \eta^{-1}\right)\right)\right. \\
& \left.\cdot H_{2} \xi^{j}\right) d \xi+C t^{1 / 2} \int_{\mathbb{R}} e^{-i t S(\xi, \eta)}\left(\chi_{1}\left(\xi \eta^{-1}\right)\right. \\
& \left.-\Theta\left(\xi \eta^{-1}\right)\right) H_{2} \xi^{j+1} \phi_{\xi}(\xi) d \xi .
\end{aligned}
$$

We find the estimates $\left|\xi \partial_{\xi}\left(\left(\chi_{1}\left(\xi \eta^{-1}\right)-\Theta\left(\xi \eta^{-1}\right)\right) H_{2} \xi^{j}\right)\right| \leq$ $C|\xi|^{j} /\left(1+t|\xi| \eta^{2}\right)$ and

$$
\begin{aligned}
& \left|\left(\chi_{1}\left(\xi \eta^{-1}\right)-\Theta\left(\xi \eta^{-1}\right)\right) H_{2} \xi^{j+1}\right| \\
& \quad+\left|\xi^{2} \partial_{\xi}\left(\left(\chi_{1}\left(\xi \eta^{-1}\right)-\Theta\left(\xi \eta^{-1}\right)\right) H_{2} \xi^{j}\right)\right| \\
& \leq \frac{C|\xi|^{1+j}}{1+t|\xi| \eta^{2}} .
\end{aligned}
$$

Then by the Hardy inequality we obtain

$$
\begin{aligned}
\left|I_{2}\right| \leq C t^{1 / 2}|\phi(0)| \int_{|\xi| \leq 3|\eta|} \frac{|\xi|^{j} d \xi}{1+t|\xi| \eta^{2}} \\
\quad+C t^{1 / 2}\left\|\partial_{\xi} \phi\right\|_{L^{2}}\left(\int_{|\xi| \leq 3|\eta|} \frac{|\xi|^{2+2 j} d \xi}{\left(1+t \xi \eta^{2}\right)^{2}}\right)^{1 / 2} .
\end{aligned}
$$

We have $\int_{|\xi| \leq 3|\eta|}\left(|\xi|^{j} d \xi /\left(1+t|\xi| \eta^{2}\right)\right) \leq C|\eta|^{j+1} \int_{0}^{3}\left(y^{j} d y /(1+\right.$ $\left.\left.t|\eta|^{3} y\right)\right) \leq C|\eta|^{j+1}\left\langle t \eta^{3}\right\rangle^{-1} \log \left\langle t \eta^{3}\right\rangle$ and $\int_{|\xi| \leq 3|\eta|}\left(|\xi|^{2+2 j} d \xi /\right.$ $\left.\left(1+t|\xi| \eta^{2}\right)^{2}\right) \leq C|\eta|^{2 j+3} \int_{0}^{3}\left(y^{2 j+2} d y /\left(1+t|\eta|^{3} y\right)^{2}\right) \leq$ $C|\eta|^{2 j+3}\left\langle t \eta^{3}\right\rangle^{-2}$. Thus we have $\left|I_{2}\right|$ s $\left.C t^{1 / 2}\left|\phi(0)\left\|\left.\eta\right|^{j+1}\left\langle t \eta^{3}\right\rangle^{-3 / 4}+C t^{1 / 2}\right\| \partial_{\xi} \phi \|_{\mathbf{L}^{2}}\right| \eta\right|^{j+3 / 2}\left\langle t \eta^{3}\right\rangle^{-1}$ for all $\geq 1, \eta \neq 0$.

To estimate $\mathscr{V}_{2} \xi^{j} \phi$ we integrate by parts via identity (24)

$$
\begin{aligned}
& \mathscr{V}_{2} \xi^{j} \phi=C t^{1 / 2} \phi(0) \\
& \cdot \int_{\mathbb{R}} e^{-i t S(\xi, \eta)} \xi \partial_{\xi}\left(H_{2} \chi_{2}\left(\xi \eta^{-1}\right) \xi^{j}\right) d \xi \\
& +C t^{1 / 2} \int_{\mathbb{R}} e^{-i t S(\xi, \eta)} \\
& . \frac{\phi(\xi)-\phi(0)}{\xi} \xi^{2} \partial_{\xi}\left(H_{2} \chi_{2}\left(\xi \eta^{-1}\right) \xi^{j}\right) d \xi \\
& +C t^{1 / 2} \int_{\mathbb{R}} e^{-i t S(\xi, \eta)} \xi^{1+j} \chi_{2}\left(\xi \eta^{-1}\right) H_{2} \phi_{\xi}(\xi) d \xi .
\end{aligned}
$$

We find the estimates $\left|\xi \partial_{\xi}\left(H_{2} \chi_{2}\left(\xi \eta^{-1}\right) \xi^{j}\right)\right| \leq C|\xi|^{j} /\left(1+t|\xi|^{3}\right)$ and $\left|\xi^{2} \partial_{\xi}\left(H_{2} \chi_{2}\left(\xi \eta^{-1}\right) \xi^{j}\right)\right|+\left|\xi^{1+j} \chi_{2}\left(\xi \eta^{-1}\right) H_{2}\right| \leq C|\xi|^{1+j} /(1+$ $\left.t|\xi|^{3}\right)$ in the domain $|\xi| \geq(3 / 2)|\eta|$. Then by the Hardy inequality we obtain

$$
\begin{aligned}
& \quad\left|\mathscr{V}_{2} \xi^{j} \phi\right| \\
& \leq C t^{1 / 2}|\phi(0)| \int_{|\xi| \geq(3 / 2)|\eta|} \frac{|\xi|^{j} d \xi}{1+t|\xi|^{3}} \\
& \quad+C t^{1 / 2}\left\|\partial_{\xi} \phi\right\|_{L^{2}}\left(\int_{|\xi| \geq(3 / 2)|\eta|} \frac{|\xi|^{2+2 j} d \xi}{\left(1+t|\xi|^{3}\right)^{2}}\right)^{1 / 2} .
\end{aligned}
$$

We have $\int_{|\xi| \geq(3 / 2)|\eta|}\left(|\xi|^{j} d \xi /\left(1+t|\xi|^{3}\right)\right) \leq C|\eta|^{1+j} \int_{3 / 2}^{\infty}\left(y^{j} d y /(1+\right.$ $\left.\left.t|\eta|^{3} y^{3}\right)\right) \leq C t^{-(j+1) / 3}\left\langle t \eta^{3}\right\rangle^{(j-2) / 3}$ and $\int_{|\xi| \geq(3 / 2)|\eta|}\left(|\xi|^{2+2 j} d \xi /(1+\right.$ $\left.\left.t|\xi|^{3}\right)^{2}\right) \leq C|\eta|^{3+2 j} \int_{3 / 2}^{\infty}\left(y^{2+2 j} d y /\left(1+t|\eta|^{3} y^{3}\right)^{2}\right) \leq$ $C t^{-2 j / 3-1}\left\langle t \eta^{3}\right\rangle^{2 j / 3-1}$ if $j=0,1$. Thus we have $\left|\mathscr{V}_{2} \xi^{j} \phi\right| \leq$ $C|\phi(0)| t^{1 / 6-j / 3}\left\langle t \eta^{3}\right\rangle^{(j-2) / 3}+C\left\|\partial_{\xi} \phi\right\|_{L^{2}} t^{-j / 3}\left\langle t \eta^{3}\right\rangle^{j / 3-1 / 2}$ for all $t \geq 1$ if $j=0$, 1 . Lemma 13 is proved.

2.3. Asymptotics for the Operator $\mathscr{V}^{*}$. We next consider the operator $\mathscr{V}^{*}$. Since $\left\|\mathscr{V}^{*} \phi\right\|_{\mathbf{L}^{\infty}} \leq C|t|^{1 / 2}\|\eta \phi\|_{\mathbf{L}^{1}}$ and $\left\|\mathscr{V}^{*} \phi\right\|_{\mathbf{L}^{2}} \leq$ $C\|\sqrt{|\eta|} \phi\|_{\mathrm{L}^{2}}$, then by the Riesz interpolation theorem (see [40], p. 52) we have

$$
\left\|\mathscr{V}^{*} \phi\right\|_{L^{p}} \leq C|t|^{1 / 2-1 / p}\left\||\eta|^{1-1 / p} \phi\right\|_{L^{p /(p-1)}}
$$

for $2 \leq p \leq \infty$. In the next lemma we find the asymptotics of $\mathscr{V}^{*}$. Denote $\hat{\xi}=\xi t^{1 / 3}$. Also define the norm $\|\phi\|_{\mathbf{I}_{\alpha, \beta}}=$ $\left\||\eta|^{\alpha}\langle\widehat{\eta}\rangle^{-\beta} \partial_{\eta} \phi\right\|_{\mathbf{L}^{2}}+\left\||\eta|^{\alpha-1}\langle\hat{\eta}\rangle^{-\beta} \phi\right\|_{\mathbf{L}^{2}}$. 
Lemma 5. Let $1 / 4+2 \beta \leq \alpha<5 / 2-2 \beta, 0 \leq \beta<1 / 2$. Then the estimate $\left\|\langle\widehat{\xi}\rangle^{\beta}\left(\mathscr{V}^{*} \phi-A^{*} \phi\right)\right\|_{\mathbf{L}^{\infty}} \leq C t^{(\alpha-1) / 3}\|\phi\|_{\mathbf{I}_{\alpha, \beta}}$ is valid for all $t \geq 1$.

Proof. We write

$$
\begin{aligned}
& \mathscr{V}^{*} \phi-A^{*} \phi \\
& =\sqrt{\frac{2|t|}{\pi}} \int_{-\infty}^{\infty} e^{i t S(\xi, \eta)}(\phi(\eta)-\phi(\xi)) \Theta\left(\eta \xi^{-1}\right)|\eta| d \eta \\
& \quad+\sqrt{\frac{2|t|}{\pi}} \int_{-\infty}^{\infty} e^{i t S(\xi, \eta)} \phi(\eta)\left(1-\Theta\left(\eta \xi^{-1}\right)\right)|\eta| d \eta \\
& =I_{1}+I_{2}
\end{aligned}
$$

for $\xi \neq 0$. In the integral $I_{1}$ we use the identity

$$
e^{i t S(\xi, \eta)}=H_{3} \partial_{\eta}\left((\eta-\xi) e^{i t S(\xi, \eta)}\right)
$$

with $H_{3}=\left(1+i t(\eta-\xi) \partial_{\eta} S(\xi, \eta)\right)^{-1}, \partial_{\eta} S(\xi, \eta)=-2|\eta|(\xi-\eta)$, and integrate by parts

$$
\begin{aligned}
I_{1} & =C t^{1 / 2} \int_{-\infty}^{\infty} e^{i t S(\xi, \eta)} \frac{\phi(\eta)-\phi(\xi)}{\eta-\xi}|\eta|^{\alpha}\langle\hat{\eta}\rangle^{-\beta}(\eta-\xi)^{2} \\
& \cdot \partial_{\eta}\left(H_{3}|\eta|^{1-\alpha}\langle\hat{\eta}\rangle^{\beta} \Theta\left(\eta \xi^{-1}\right)\right) d \eta \\
& +C t^{1 / 2} \int_{-\infty}^{\infty} e^{i t S(\xi, \eta)}(\eta-\xi) H_{3}|\eta|^{1-\alpha}\langle\hat{\eta}\rangle^{\beta} \Theta\left(\eta \xi^{-1}\right) \\
& \cdot \partial_{\eta}\left(|\eta|^{\alpha}\langle\hat{\eta}\rangle^{-\beta} \phi(\eta)\right) d \eta .
\end{aligned}
$$

Then apply the estimates $\left.\left|(\eta-\xi) H_{3}\right| \eta\right|^{1-\alpha}\langle\widehat{\eta}\rangle^{\beta} \Theta\left(\eta \xi^{-1}\right)|+|(\eta-$ $\xi)\left.^{2}\langle\widehat{\eta}\rangle^{\beta} \partial_{\eta}\left(H_{3}|\eta|^{1-\alpha} \Theta\left(\eta \xi^{-1}\right)\right)|\leq C| \xi\right|^{1-\alpha}\langle\widehat{\xi}\rangle^{\beta}|\eta-\xi| /(1+t|\xi|(\eta-$ $\left.\xi)^{2}\right)$ in the domain $1 / 3 \leq \eta / \xi \leq 3$. If $|\kappa(x)| \leq C|\kappa(y x)|$ for all $y \in(0,1)$ then we find the Hardy inequality

$$
\begin{aligned}
& \left\|\frac{\kappa(x)}{x}(\phi(x)-\phi(0))\right\|_{\mathbf{L}^{2}}=\left\|\frac{\kappa(x)}{x} \int_{0}^{x} \phi^{\prime}(y) d t y\right\|_{\mathbf{L}^{2}} \\
& =\left\|\kappa(x) \int_{0}^{1} \phi^{\prime}(x y) d y\right\|_{\mathbf{L}^{2}} \\
& \quad \leq C\left\|\int_{0}^{1} \kappa(x y) \phi^{\prime}(x y) d y\right\|_{L^{2}} \\
& \quad \leq \int_{0}^{1}\left\|\kappa(x y) \phi^{\prime}(x y)\right\|_{\mathbf{L}^{2}} d t \leq \int_{0}^{1} y^{-1 / 2} d y\left\|\kappa \phi^{\prime}\right\|_{\mathbf{L}^{2}} \\
& \quad \leq 2\left\|\kappa \phi^{\prime}\right\|_{\mathbf{L}^{2}} .
\end{aligned}
$$

Hence $\int_{1 / 3 \leq \eta / \xi \leq 3}\left(|\phi(\eta)-\phi(\xi)|^{2} /(\eta-\xi)^{2}\right)|\eta|^{2 \alpha}\langle\widehat{\eta}\rangle^{-2 \beta} d \eta \leq$ $C\|\phi\|_{\mathbf{I}_{\alpha, \beta}}^{2}$. Therefore changing $\eta=y \xi$, we have

$$
\begin{aligned}
& \langle\hat{\xi}\rangle^{\beta}\left|I_{1}\right| \leq C t^{1 / 2}|\xi|^{1-\alpha}\langle\hat{\xi}\rangle^{2 \beta}\|\phi\|_{\mathbf{I}_{\alpha, \beta}} \\
& \cdot\left(\int_{1 / 3 \leq \eta / \xi \leq 3} \frac{(\eta-\xi)^{2} d \eta}{\left(1+t|\xi|(\eta-\xi)^{2}\right)^{2}}\right)^{1 / 2} \\
& \leq C t^{1 / 2}|\xi|^{1-\alpha}\langle\widehat{\xi}\rangle^{2 \beta}\|\phi\|_{\mathbf{I}_{\alpha, \beta}} \\
& \cdot\left(\int_{1 / 3}^{3} \frac{|\xi|^{3}(1-y)^{2} d y}{\left(1+t|\xi|^{3}(1-y)^{2}\right)^{2}}\right)^{1 / 2} \leq C t^{1 / 2}|\xi|^{5 / 2-\alpha} \\
& \cdot\langle\hat{\xi}\rangle^{2 \beta-9 / 4}\|\phi\|_{\mathbf{I}_{\alpha, \beta}} \leq C t^{(\alpha-1) / 3}\|\phi\|_{\mathbf{I}_{\alpha, \beta}}
\end{aligned}
$$

if $1 / 4+2 \beta \leq \alpha \leq 5 / 2$. In the integral $I_{2}$ using the identity

$$
e^{i t S(\xi, \eta)}=H_{4} \partial_{\eta}\left(\eta e^{i t S(\xi, \eta)}\right)
$$

with $H_{4}=\left(1+i t \eta \partial_{\eta} S(\xi, \eta)\right)^{-1}, \partial_{\eta} S(\xi, \eta)=-2|\eta|(\xi-\eta)$, we integrate by parts

$$
\begin{aligned}
I_{2} & =C t^{1 / 2} \int_{-\infty}^{\infty} e^{i t S(\xi, \eta)} \\
& \cdot \frac{|\eta|^{\alpha} \phi(\eta)}{\eta\langle\hat{\eta}\rangle^{\beta}} \eta^{2} \partial_{\eta}\left(H_{4}\left(1-\Theta\left(\eta \xi^{-1}\right)\right)|\eta|^{1-\alpha}\right. \\
& \left.\cdot\langle\widehat{\eta}\rangle^{\beta}\right) d \eta+C t^{1 / 2} \int_{-\infty}^{\infty} e^{i t S(\xi, \eta)} \eta H_{4}(1 \\
& \left.-\Theta\left(\eta \xi^{-1}\right)\right)|\eta|^{1-\alpha}\langle\widehat{\eta}\rangle^{\beta} \partial_{\eta}\left(|\eta|^{\alpha}\langle\widehat{\eta}\rangle^{-\beta} \phi(\eta)\right) d \eta .
\end{aligned}
$$

Then using the estimates $\partial_{\eta} S(\xi, \eta)=O(|\eta|(\xi+|\eta|))$ in the domains $\eta / \xi<1 / 3$ and $\eta / \xi \geq 3$, we get

$$
\begin{aligned}
\mid \eta^{2} \partial_{\eta} & \left(H_{4}\left(1-\Theta\left(\eta \xi^{-1}\right)\right)|\eta|^{1-\alpha}\langle\hat{\eta}\rangle^{\beta}\right) \mid \\
& +\left.\left|\eta H_{4}\left(1-\Theta\left(\eta \xi^{-1}\right)\right)\right| \eta\right|^{1-\alpha}\langle\hat{\eta}\rangle^{\beta} \mid \\
\leq & \frac{C|\eta|^{2-\alpha}\langle\widehat{\eta}\rangle^{\beta}}{1+t \eta^{2}(|\xi|+|\eta|)} .
\end{aligned}
$$

Therefore by the Hardy inequality

$$
\begin{gathered}
\langle\hat{\xi}\rangle^{\beta}\left|I_{2}\right| \leq C t^{1 / 2}\langle\hat{\xi}\rangle^{\beta}\left\|\partial_{\eta}\left(|\eta|^{\alpha}\langle\hat{\eta}\rangle^{-\beta} \phi(\eta)\right)\right\|_{\mathbf{L}^{2}} \\
\cdot\left(\int_{-\infty}^{\infty} \frac{\langle\hat{\eta}\rangle^{2 \beta}|\eta|^{4-2 \alpha} d \eta}{\left(1+t \eta^{2}(|\xi|+|\eta|)\right)^{2}}\right)^{1 / 2} \leq C t^{1 / 2}\|\phi\|_{\mathbf{I}_{\alpha, \beta}} \\
\cdot\left(\int_{-\infty}^{\infty} \frac{\langle\hat{\xi}\rangle^{2 \beta}\langle\hat{\eta}\rangle^{2 \beta}|\eta|^{4-2 \alpha} d \eta}{\left(1+t \eta^{2}(|\xi|+|\eta|)\right)^{2}}\right)^{1 / 2} \cdot
\end{gathered}
$$


We have

$$
\begin{aligned}
& \int_{-\infty}^{\infty} \frac{\langle\widehat{\xi}\rangle^{2 \beta}\langle\widehat{\eta}\rangle^{2 \beta}|\eta|^{4-2 \alpha} d \eta}{\left(1+t \eta^{2}(|\xi|+|\eta|)\right)^{2}} \\
& \leq C \int_{0}^{1} \frac{\langle\widehat{\xi}\rangle^{2 \beta} \eta^{4-2 \alpha}\langle\widehat{\eta}\rangle^{2 \beta} d \eta}{\left(1+t \eta^{2}(|\xi|+|\eta|)\right)^{2}} \\
& +C t^{2 \beta / 3-2} \int_{1}^{\infty}\langle\widehat{\xi}\rangle^{2 \beta} \frac{\eta^{2 \beta-2 \alpha} d \eta}{(|\xi|+|\eta|)^{2}} \\
& \leq C \int_{0}^{1} \eta^{4-2 \alpha}\langle\widehat{\eta}\rangle^{2 \beta-6} d \eta \\
& +C t^{-4 \beta / 3} \int_{0}^{1} \eta^{4-4 \beta-2 \alpha}\langle\widehat{\eta}\rangle^{6 \beta-6} d \eta \\
& +C t^{4 \beta / 3-2} \int_{1}^{\infty} \eta^{4 \beta-2 \alpha-2} d \eta \\
& \leq C t^{(2 \alpha-5) / 3} \int_{0}^{t^{1 / 3}} \eta^{4-2 \alpha}\langle\eta\rangle^{2 \beta-6} d \eta \\
& +C t^{(2 \alpha-5) / 3} \int_{0}^{t^{1 / 3}} \eta^{4-4 \beta-2 \alpha}\langle\eta\rangle^{6 \beta-6} d \eta+C t^{4 \beta / 3-2} \\
& \leq C t^{(2 \alpha-5) / 3}
\end{aligned}
$$

if $1 / 4+2 \beta \leq \alpha<5 / 2-2 \beta, 0 \leq \beta<1 / 2$. Therefore we get $\langle\widehat{\xi}\rangle^{\beta}\left|I_{2}\right| \leq C t^{(\alpha-1) / 3}\|\phi\|_{\mathbf{I}_{\alpha, \beta}}$. Lemma 5 is proved.

\section{Commutators with $\mathscr{V}$}

First we estimate the Fourier type integral

$$
\mathscr{W} \phi=t^{1 / 2} \int_{\mathbb{R}} e^{-i t S(\xi, \eta)} q(t, \xi, \eta) \phi(\xi) d \xi
$$

in the $\mathbf{L}^{2}$-norm. In the particular factorized case $q(t, \xi, \eta)=$ $q_{1}(\xi) q_{2}(\eta)$, with estimate $\left|q_{2}(\mu(x))\right| \leq|\mu(x)|^{1 / 2}$, we find

$$
\begin{aligned}
& \|\mathscr{W} \phi\|_{\mathbf{L}^{2}} \leq t^{1 / 2} \| \bar{M} q_{2}(\eta) \\
& \cdot \int_{\mathbb{R}} e^{i t \eta^{2} \xi} e^{-(i t / 3)|\xi|^{3}} q_{1}(\xi) \phi(\xi) d \xi \|_{\mathbf{L}^{2}} \\
& \leq t^{1 / 2} \| q_{2}(\mu(x))|\mu(x)|^{-1 / 2} \\
& \cdot \int_{\mathbb{R}} e^{i t x \xi} e^{-(i t / 3)|\xi|^{3}} q_{1}(\xi) \phi(\xi) d \xi \|_{\mathbf{L}^{2}} \\
& \leq C\left\|e^{-(i t / 3)|\xi|^{3}} q_{1} \phi\right\|_{\mathbf{L}^{2}}=C\left\|q_{1} \phi\right\|_{\mathbf{L}^{2}} .
\end{aligned}
$$

Next we obtain a more general result.

Lemma 6. Suppose that $\left|\left(\eta \partial_{\eta}\right)^{k} q(t, \xi, \eta)\right| \leq C\{\widehat{\eta}\}^{v}\langle\widehat{\eta}\rangle^{-v}$ for all $\xi, \eta \in \mathbb{R}, k=0,1,2$, where $v \in(0,1)$. Then the estimate $\left\||\eta|^{1 / 2} \mathscr{W} \phi\right\|_{\mathrm{L}^{2}} \leq C\|\phi\|_{\mathrm{L}^{2}}$ is true for all $t \geq 1$.
Proof. We write

$$
\begin{aligned}
& \left\||\eta|^{1 / 2} \mathscr{W} \phi\right\|_{L^{2}}^{2}=C t \int_{\mathbb{R}} d \eta|\eta| \int_{\mathbb{R}} d \xi \\
& \cdot \int_{\mathbb{R}} d \zeta e^{i t(S(\zeta, \eta)-S(\xi, \eta))} q(t, \xi, \eta) \phi(\xi) \overline{q(t, \zeta, \eta)} \overline{\phi(\zeta)} \\
& =C t \int_{\mathbb{R}} d \xi e^{-(i t / 3)|\xi|^{3}} \phi(\xi) \\
& \cdot \int_{\mathbb{R}} d \zeta e^{(i t / 3)|\zeta|^{3}} \overline{\phi(\zeta)} K(t, \xi, \zeta),
\end{aligned}
$$

where the kernel $K(t, \xi, \zeta)=\int_{\mathbb{R}} d \eta|\eta| e^{i t \eta|\eta|(\xi-\zeta)} q(t, \xi, \eta) \overline{q(t, \zeta, \eta)}$. Integrating two times by parts via the identity $e^{i t \eta|\eta|(\xi-\zeta)}=$ $H \partial_{\eta}\left(\eta e^{i t \eta|\eta|(\xi-\zeta)}\right)$, with $H=(1+2 i t \eta|\eta|(\xi-\zeta))^{-1}$ we get

$$
\begin{aligned}
& K(t, \xi, \zeta)=\int_{\mathbb{R}} e^{i t \eta^{2}(\xi-\zeta)} \eta \partial_{\eta}\left(H \eta \partial_{\eta}(H|\eta| q(t, \xi, \eta)\right. \\
& \cdot \overline{q(t, \zeta, \eta)})) d \eta
\end{aligned}
$$

Since $\left|\eta \partial_{\eta}\left(H \eta \partial_{\eta}(H|\eta| q(t, \xi, \eta) \overline{q(t, \zeta, \eta)})\right)\right| \leq C|\eta|\{\widehat{\eta}\}^{2 v}\langle\widehat{\eta}\rangle^{-2 v} /$ $\left(1+t \eta^{2}|\xi-\zeta|\right)^{2}$ we get

$$
\begin{aligned}
& |K(t, \xi, \zeta)| \\
& \leq C \int_{0}^{1} \frac{\{\hat{\eta}\}^{2 v}\langle\hat{\eta}\rangle^{-2 v}|\eta| d \eta}{\left(1+t \eta^{2}|\xi-\zeta|\right)^{2}} \\
& \quad+C \int_{1}^{\infty} \frac{\langle\hat{\eta}\rangle^{-2 v}|\eta| d \eta}{\left(1+t \eta^{2}|\xi-\zeta|\right)^{2}} \\
& \leq C t^{-2 / 3} \int_{0}^{1} \frac{\eta^{1+2 v} d \eta}{\left(1+\eta^{2} t^{1 / 3}|\xi-\zeta|\right)^{2}} \\
& \quad+C t^{-2 / 3} \int_{1}^{\infty} \frac{\eta^{1-2 v} d \eta}{\left(1+t^{1 / 3} \eta^{2}|\xi-\zeta|\right)^{2}} \\
& \quad+C t^{-2 v / 3}(|\xi-\zeta| t)^{\nu-1} \int_{t^{1 / 2}|\xi-\zeta|^{1 / 2}}^{\infty} \eta^{1-2 v}\langle\eta\rangle^{-4} d \eta \\
& \leq C t^{-2 / 3}\left\langle(\xi-\zeta) t^{1 / 3}\right\rangle^{-1-v} \\
& \quad+C t^{-2 / 3}\left(|\xi-\zeta| t^{1 / 3}\right)^{\nu-1}\left\langle(\xi-\zeta) t^{1 / 3}\right\rangle \\
& \quad+C t^{-2 v / 3}(|\xi-\zeta| t)^{\nu-1}\langle(\xi-\zeta) t\rangle^{-1-\nu} \\
& \quad
\end{aligned}
$$

Then by the Cauchy-Schwarz inequality and Young inequality we obtain

$$
\begin{aligned}
& \left\||\eta|^{1 / 2} \mathscr{W} \phi\right\|_{\mathbf{L}^{2}}^{2} \leq C t^{1 / 3}\|\phi\|_{\mathbf{L}^{2}} \\
& \cdot\left\|\int\left\langle(\xi-\zeta) t^{1 / 3}\right\rangle^{-1-\gamma}|\phi(\zeta)| d \zeta\right\|_{\mathbf{L}^{2}}+C t^{1 / 3}\|\phi\|_{\mathbf{L}^{2}} \\
& \cdot\left\|\int\left(|\xi-\zeta| t^{1 / 3}\right)^{\nu-1}\left\langle(\xi-\zeta) t^{1 / 3}\right\rangle^{-1}|\phi(\zeta)| d \zeta\right\|_{\mathbf{L}^{2}} \\
& +C t^{1-2 v / 3}\|\phi\|_{\mathbf{L}^{2}}
\end{aligned}
$$




$$
\begin{aligned}
& \cdot\left\|\int(|\xi-\zeta| t)^{\nu-1}\langle(\xi-\zeta) t\rangle^{-1-\gamma}|\phi(\zeta)| d \zeta\right\|_{\mathbf{L}^{2}} \\
& \leq C t^{1 / 3}\left\|\left\langle\xi t^{1 / 3}\right\rangle^{-1-\nu}\right\|_{\mathbf{L}^{1}}\|\phi\|_{\mathbf{L}^{2}}^{2} \\
& +C t^{1 / 3}\left\|\left(|\xi| t^{1 / 3}\right)^{\nu-1}\left\langle\xi t^{1 / 3}\right\rangle^{-1}\right\|_{\mathbf{L}^{1}}\|\phi\|_{\mathbf{L}^{2}}^{2} \\
& +C t^{1-2 v / 3}\left\|(|\xi| t)^{\nu-1}\langle\xi t\rangle^{-1-\nu}\right\|_{\mathbf{L}^{1}}\|\phi\|_{\mathbf{L}^{2}}^{2} \leq C\|\phi\|_{\mathbf{L}^{2}}^{2}
\end{aligned}
$$

if $\nu \in(0,1)$. Lemma 14 is proved.

Next we estimate $\mathscr{W} \phi(0)$.

Lemma 7. Suppose that $\left|\left(\xi \partial_{\xi}\right)^{k} q(t, \xi, \eta)\right| \leq C(|\eta|+|\xi|)^{-1}$ for all $\xi, \eta \in \mathbb{R}, k=0,1$. Then the estimate $\left\||\eta|^{1 / 2} \mathscr{W} 1\right\|_{\mathbf{L}^{2}} \leq C t^{1 / 6}$ is true.

Proof. As in the proof of Lemma 13 we decompose

$$
\begin{aligned}
\mathscr{W} 1 & =t^{1 / 2} \int_{\mathbb{R}} e^{-i t S(\xi, \eta)} q(t, \xi, \eta) d \xi \\
= & t^{1 / 2} \int_{\mathbb{R}} e^{-i t S(\xi, \eta)} q(t, \xi, \eta) \Theta\left(\xi \eta^{-1}\right) d \xi \\
& +t^{1 / 2} \int_{\mathbb{R}} e^{-i t S(\xi, \eta)} q(t, \xi, \eta) \\
& +\left(\chi_{1}\left(\xi \eta^{-1}\right)-\Theta\left(\xi \eta^{-1}\right)\right) d \xi \\
& +t^{1 / 2} \int_{\mathbb{R}} e^{-i t S(\xi, \eta)} q(t, \xi, \eta) \chi_{2}\left(\xi \eta^{-1}\right) d \xi=I_{1} \\
& +I_{2}+I_{3}
\end{aligned}
$$

for $\eta \neq 0$. In the first summand $I_{1}$ we integrate by parts via identity (19), to get

$$
\begin{aligned}
I_{1}= & C t^{1 / 2} \int_{\mathbb{R}} e^{-i t S(\xi, \eta)}(\xi-\eta) \\
& \cdot \partial_{\xi}\left(H_{1} q(t, \xi, \eta) \Theta\left(\xi \eta^{-1}\right)\right) d \xi .
\end{aligned}
$$

Using the estimate $\left|(\xi-\eta) \partial_{\xi}\left(H_{1} q(t, \xi, \eta) \Theta\left(\xi \eta^{-1}\right)\right)\right| \leq$ $C|\eta|^{-1} /\left(1+t|\eta|(\xi-\eta)^{2}\right)$ then changing $\xi=\eta y$, we obtain

$$
\begin{aligned}
\left|I_{1}\right| & \leq C t^{1 / 2}|\eta|^{-1} \int_{1 / 3<\xi / \eta<3} \frac{d \xi}{1+t|\eta|(\xi-\eta)^{2}} \\
& \leq C t^{1 / 2} \int_{1 / 3}^{3} \frac{d y}{1+t|\eta|^{3}(y-1)^{2}} \leq C t^{1 / 2}\langle\widehat{\eta}\rangle^{-3 / 2} .
\end{aligned}
$$

Thus we get $\left\||\eta|^{1 / 2} I_{1}\right\|_{\mathbf{L}^{2}} \leq C t^{1 / 2}\left\||\eta|^{1 / 2}\langle\widehat{\eta}\rangle^{-3 / 2}\right\|_{\mathbf{L}^{2}} \leq C t^{1 / 6}$. To estimate the integral $I_{2}$ we integrate by parts via identity (24), to get

$$
\begin{aligned}
I_{2}= & C t^{1 / 2} \int_{\mathbb{R}} e^{-i t S(\xi, \eta)} \xi \partial_{\xi}\left(\left(\chi_{1}\left(\xi \eta^{-1}\right)-\Theta\left(\xi \eta^{-1}\right)\right)\right. \\
& \left.\cdot H_{2} q(t, \xi, \eta)\right) d \xi
\end{aligned}
$$

We find the estimates $\left|\xi \partial_{\xi}\left(\left(\chi_{1}\left(\xi \eta^{-1}\right)-\Theta\left(\xi \eta^{-1}\right)\right) H_{2} q(t, \xi, \eta)\right)\right| \leq$ $C|\eta|^{-1} /\left(1+t|\xi| \eta^{2}\right)$, then we obtain

$$
\begin{aligned}
\left|I_{2}\right| & \leq C t^{1 / 2}|\eta|^{-1} \int_{|\xi| \leq 3|\eta|} \frac{d \xi}{1+t|\xi| \eta^{2}} \\
& \leq C t^{1 / 2} \int_{0}^{3} \frac{d y}{1+t|\eta|^{3} y} \leq C t^{1 / 2}\langle\widehat{\eta}\rangle^{-3 / 2} .
\end{aligned}
$$

Thus as above we get $\left\||\eta|^{1 / 2} I_{2}\right\|_{\mathrm{L}^{2}} \leq C t^{1 / 6}$ for all $t \geq 1$ if $v>$ $-1 / 2$. To estimate $I_{3}$ we integrate by parts via identity (24)

$$
I_{3}=C t^{1 / 2} \int_{\mathbb{R}} e^{-i t S(\xi, \eta)} \xi \partial_{\xi}\left(H_{2} \chi_{2}\left(\xi \eta^{-1}\right) q(t, \xi, \eta)\right) d \xi .
$$

We find the estimates $\left|\xi \partial_{\xi}\left(H_{2} \chi_{2}\left(\xi \eta^{-1}\right) q(t, \xi, \eta)\right)\right| \leq C /(|\eta|+$ $|\xi|)\left(1+t|\xi|^{3}\right)$ in the domain $|\xi| \geq(3 / 2)|\eta|$, and then we obtain

$$
\begin{aligned}
\left|I_{3}\right| \leq C t^{1 / 2} \int_{(3 / 2)|\eta|}^{\infty} \frac{d \xi}{(|\eta|+|\xi|)\left(1+t|\xi|^{3}\right)} \\
\leq C t^{1 / 2} \int_{3 / 2}^{(3 / 2)\langle\eta\rangle|\eta|^{-1}} \frac{d y}{(1+y)\left(1+t|\eta|^{3} y^{3}\right)} \\
\quad+C t^{-1 / 2}\langle\eta\rangle^{-1} \int_{(3 / 2)\langle\eta\rangle}^{\infty} \xi^{-3} d \xi \\
\leq C t^{1 / 2}|\hat{\eta}|^{-\gamma}\langle\hat{\eta}\rangle^{\gamma-3}+C t^{-1 / 2}\langle\eta\rangle^{-3}
\end{aligned}
$$

if $0<\gamma<1$. Thus we find

$$
\begin{aligned}
\left\||\eta|^{1 / 2} I_{3}\right\|_{\mathbf{L}^{2}} \leq & C t^{1 / 2}\left\||\eta|^{1 / 2}|\hat{\eta}|^{-\gamma}\langle\widehat{\eta}\rangle^{\gamma-3}\right\|_{\mathbf{L}^{2}} \\
& +C t^{-1 / 2}\left\|\langle\eta\rangle^{-5 / 2}\right\|_{\mathbf{L}^{2}} \leq C t^{1 / 6}
\end{aligned}
$$

for all $t \geq 1$. Lemma 7 is proved.

In the next lemma we estimate the commutator $\left[\eta, \mathscr{V}_{1}\right]$. Define the norm $\|\phi\|_{\mathbf{Y}}=\|\phi\|_{\mathbf{L}^{\infty}}+t^{-1 / 6}\left\|\partial_{\xi} \phi\right\|_{\mathbf{L}^{2}}$.

Lemma 8. Let $j \geq 0, v \in(0,1)$. Then the estimate $\left\||\eta|^{3 / 2-j}\{\hat{\eta}\}^{v}\langle\hat{\eta}\rangle^{-v} t\left[\eta, \mathscr{V}_{1}\right] \xi^{j} \phi\right\|_{L^{2}} \leq C t^{1 / 6}\|\phi\|_{\mathbf{Y}}$ is true for all $t \geq 1$.

Proof. For $\eta \neq 0$ we integrate by parts

$$
\begin{gathered}
|\eta|^{1-j}\{\hat{\eta}\}^{\nu}\langle\widehat{\eta}\rangle^{-v} t\left[\eta, \mathscr{V}_{1}\right] \xi^{j} \phi=|\eta|^{1-j}\{\hat{\eta}\}^{\nu}\langle\widehat{\eta}\rangle^{-\nu} \\
\cdot \sqrt{\frac{|t|}{2 \pi}} \int_{\mathbb{R}} e^{-i t S(\xi, \eta)} \partial_{\xi}\left(\frac{\eta-\xi}{i \partial_{\xi} S(\xi, \eta)} \chi_{1}\left(\xi \eta^{-1}\right)\right. \\
\left.\cdot \xi^{j} \phi(\xi)\right) d \xi=\sqrt{\frac{|t|}{2 \pi}} \int_{\mathbb{R}} e^{-i t S(\xi, \eta)} q_{1}(t, \xi, \eta)
\end{gathered}
$$




$$
\begin{aligned}
& \cdot \partial_{\xi} \phi(\xi) d \xi+\sqrt{\frac{|t|}{2 \pi}} \int_{\mathbb{R}} e^{-i t S(\xi, \eta)} q_{2}(t, \xi, \eta) \\
& \cdot \frac{\phi(\xi)-\phi(0)}{\xi} d \xi+\phi(0) \\
& \cdot \sqrt{\frac{|t|}{2 \pi}} \int_{\mathbb{R}} e^{-i t S(\xi, \eta)} q_{3}(t, \xi, \eta) d \xi
\end{aligned}
$$

where $q_{1}(t, \xi, \eta)=\{\hat{\eta}\}^{\nu}\langle\hat{\eta}\rangle^{-v}|\eta|^{1-j}\left((\eta-\xi) / i \partial_{\xi} S(\xi\right.$, $\eta)) \xi^{j} \chi_{1}\left(\xi \eta^{-1}\right), q_{2}(t, \xi, \eta)=\xi \partial_{\xi} q_{1}(t, \xi, \eta)$, and $q_{3}(t, \xi, \eta)=$ $\partial_{\xi} q_{1}(t, \xi, \eta)$. Since $\partial_{\xi} S(\xi, \eta)=\xi|\xi|-\eta|\eta|$ and $(\eta-\xi) /$ $\partial_{\xi} S(\xi, \eta)=(|\xi|+|\eta|) /\left(\xi^{2}+\eta^{2}+|\xi||\eta|+\eta \xi\right)$, we have

$$
\begin{aligned}
q_{1}(t, \xi, \eta) & =|\eta|^{1-j}\{\hat{\eta}\}^{\nu}\langle\widehat{\eta}\rangle^{-\nu} \frac{\eta-\xi}{\xi|\xi|-\eta|\eta|} \xi^{j} \chi_{1}\left(\xi \eta^{-1}\right) \\
& =O\left(\{\hat{\eta}\}^{\nu}\langle\hat{\eta}\rangle^{-v}\right)
\end{aligned}
$$

and similarly $q_{2}(t, \xi, \eta)=\xi \partial_{\xi} q_{1}(\xi, \eta)=O\left(\{\hat{\eta}\}^{\nu}\langle\hat{\eta}\rangle^{-v}\right)$ for all $\xi, \eta \in \mathbb{R}$ in the domain $|\xi| \leq 3|\eta|$. Hence we have $\left|\left(\eta \partial_{\eta}\right)^{k} q_{l}(t, \xi, \eta)\right| \leq C\{\hat{\eta}\}^{\nu}\langle\hat{\eta}\rangle^{-v}$ for all $\xi, \eta \in \mathbb{R}, k=0,1,2$, and $l=1,2$, where $v \in(0,1)$, and by Lemma 14

$$
\begin{aligned}
& \left\||\eta|^{1 / 2} \sqrt{\frac{|t|}{2 \pi}} \int_{\mathbb{R}} e^{-i t S(\xi, \eta)} q_{1}(t, \xi, \eta) \partial_{\xi} \phi(\xi) d \xi\right\|_{L^{2}} \\
& \leq C\left\|\partial_{\xi} \phi\right\|_{\mathbf{L}^{2}}
\end{aligned}
$$

and by the Hardy inequality and Lemma 14

$$
\begin{aligned}
& \left\||\eta|^{1 / 2} \sqrt{\frac{|t|}{2 \pi}} \int_{\mathbb{R}} e^{-i t S(\xi, \eta)} q_{2}(t, \xi, \eta) \frac{\phi(\xi)-\phi(0)}{\xi} d \xi\right\|_{\mathbf{L}^{2}} \\
& \leq C\left\|\frac{\phi(\xi)-\phi(0)}{\xi}\right\|_{\mathbf{L}^{2}} \leq C\left\|\partial_{\xi} \phi\right\|_{\mathbf{L}^{2}} .
\end{aligned}
$$

Also we have

$$
\begin{aligned}
q_{3} & (t, \xi, \eta) \\
& =|\eta|^{1-j}\{\widehat{\eta}\}^{\nu}\langle\widehat{\eta}\rangle^{-v} \partial_{\xi}\left(\frac{\eta-\xi}{(\xi|\xi|-\eta|\eta|)} \xi^{j} \chi_{1}\left(\xi \eta^{-1}\right)\right) \\
& =O\left((|\eta|+|\xi|)^{-1}\langle\eta\rangle^{\beta+j-1}\right)
\end{aligned}
$$

for all $\xi, \eta \in \mathbb{R}$, in the domain $|\xi| \leq 3|\eta|$. Hence we get $\left|\left(\xi \partial_{\xi}\right)^{k} q_{3}(t, \xi, \eta)\right| \leq C(|\eta|+|\xi|)^{-1}$ for all $\xi, \eta \in$ $\mathbb{R}, k=0,1$. Therefore applying Lemma 7 we obtain $\left\||\eta|^{1 / 2} \sqrt{|t| / 2 \pi} \int_{\mathbb{R}} e^{-i t S(\xi, \eta)} q_{3}(t, \xi, \eta) d \xi\right\|_{\mathbf{L}^{2}} \leq C^{1 / 6}$. Lemma 8 is proved.

In the next lemma we estimate the operator $\mathscr{V}_{2}$.

Lemma 9. Let $j=0,1,2, v \in(0,1)$. Then the estimate $\left\||\eta|^{5 / 2-j}\{\widehat{\eta}\}^{v}\langle\widehat{\eta}\rangle^{-v} t \mathscr{V}_{2} \xi^{j} \phi\right\|_{\mathbf{L}^{2}} \leq C t^{1 / 6}\|\phi\|_{\mathrm{Y}}$ is true for all $t \geq 1$.
Proof. We integrate by parts

$$
\begin{aligned}
& \{\hat{\eta}\}^{\nu}\langle\hat{\eta}\rangle^{-v}|\eta|^{2-j} t \mathscr{V}_{2} \xi^{j} \phi=\{\widehat{\eta}\}^{\nu}\langle\widehat{\eta}\rangle^{-v}|\eta|^{2-j} \\
& \cdot \sqrt{\frac{|t|}{2 \pi}} \int_{\mathbb{R}} e^{-i t S(\xi, \eta)} \partial_{\xi}\left(\frac{\xi^{j} \chi_{2}\left(\xi \eta^{-1}\right)}{i \partial_{\xi} S(\xi, \eta)} \phi(\xi)\right) d \xi \\
& \quad=\sqrt{\frac{|t|}{2 \pi}} \int_{\mathbb{R}} e^{-i t S(\xi, \eta)} q_{1}(t, \xi, \eta) \partial_{\xi} \phi(\xi) d \xi \\
& \quad+\sqrt{\frac{|t|}{2 \pi}} \int_{\mathbb{R}} e^{-i t S(\xi, \eta)} q_{2}(t, \xi, \eta) \frac{\phi(\xi)-\phi(0)}{\xi} d \xi \\
& \quad+\phi(0) \sqrt{\frac{|t|}{2 \pi}} \int_{\mathbb{R}} e^{-i t S(\xi, \eta)} q_{3}(t, \xi, \eta) d \xi,
\end{aligned}
$$

where $q_{1}(t, \xi, \eta)=\left(\{\hat{\eta}\}^{\nu}\langle\widehat{\eta}\rangle^{-v}|\eta|^{2-j} \xi^{j} / i \partial_{\xi} S(\xi, \eta)\right) \chi_{2}\left(\xi \eta^{-1}\right)$, $q_{2}(t, \xi, \eta)=\xi \partial_{\xi} q_{1}(t, \xi, \eta)$, and $q_{3}(t, \xi, \eta)=\partial_{\xi} q_{1}(t, \xi, \eta)$. We find

$$
\begin{aligned}
q_{1}(t, \xi, \eta) & =O\left(\{\hat{\eta}\}^{\nu}\langle\widehat{\eta}\rangle^{-v}|\eta|^{2-j}|\xi|^{j-2}\right) \\
& =O\left(\{\hat{\eta}\}^{\nu}\langle\widehat{\eta}\rangle^{-v}\right),
\end{aligned}
$$

and $q_{2}(t, \xi, \eta)=O\left(\{\hat{\eta}\}^{\nu}\langle\hat{\eta}\rangle^{-v}\right)$ for all $\xi, \eta \in \mathbb{R}$, in the domain $|\xi| \geq(3 / 2)|\eta|$ if $j=0,1,2$. Then $\left|\left(\eta \partial_{\eta}\right)^{k} q_{l}(t, \xi, \eta)\right| \leq$ $C\{\widehat{\eta}\}^{v}\langle\widehat{\eta}\rangle^{-v}$ for all $\xi, \eta \in \mathbb{R}, k=0,1,2$, and $l=1,2$. Hence by Lemma 14 we find

$$
\begin{aligned}
& \left\||\eta|^{1 / 2} \sqrt{\frac{|t|}{2 \pi}} \int_{\mathbb{R}} e^{-i t S(\xi, \eta)} q_{1}(t, \xi, \eta) \partial_{\xi} \phi(\xi) d \xi\right\|_{\mathbf{L}^{2}} \\
& \leq C\left\|\partial_{\xi} \phi\right\|_{\mathbf{L}^{2}},
\end{aligned}
$$

and by the Hardy inequality

$$
\begin{aligned}
& \left\||\eta|^{1 / 2} \sqrt{\frac{|t|}{2 \pi}} \int_{\mathbb{R}} e^{-i t S(\xi, \eta)} q_{2}(t, \xi, \eta) \frac{\phi(\xi)-\phi(0)}{\xi} d \xi\right\|_{L^{2}} \\
& \leq C\left\|\frac{\phi(\xi)-\phi(0)}{\xi}\right\|_{L^{2}} \leq C\left\|\partial_{\xi} \phi\right\|_{L^{2}} .
\end{aligned}
$$

Also we have $q_{3}(t, \xi, \eta)=O\left((|\eta|+|\xi|)^{-1}\right)$ for all $\xi, \eta \in \mathbb{R}$, in the domain $|\xi| \geq(3 / 2)|\eta|$, if $j=0,1,2$. Hence $\left|\left(\xi \partial_{\xi}\right)^{k} q_{3}(t, \xi, \eta)\right| \leq$ $C(|\eta|+|\xi|)^{-1}$ for all $\xi, \eta \in \mathbb{R}, k=0,1$, and by Lemma 7

$$
\left\||\eta|^{1 / 2} \sqrt{\frac{|t|}{2 \pi}} \int_{\mathbb{R}} e^{-i t S(\xi, \eta)} q_{3}(t, \xi, \eta) d \xi\right\|_{\mathbf{L}^{2}} \leq C t^{1 / 6} .
$$

Lemma 9 is proved.

In the next lemma, we estimate the derivative $\partial_{\eta} \mathscr{V}$.

Lemma 10. Let $j=0,1, v \in(0,1)$. Then the estimate $\left\||\eta|^{1 / 2-j}\{\widehat{\eta}\}^{v}\langle\widehat{\eta}\rangle^{-v} \partial_{\eta} \mathscr{V} \xi^{j} \phi\right\|_{\mathbf{L}^{2}} \leq C t^{1 / 6}\|\phi\|_{\mathbf{Y}}$ is true for all $t \geq 1$. 
Proof. Since $\mathscr{A}_{1} \mathscr{V}=\mathscr{V} i \xi$ with $\mathscr{A}_{1}=\mathscr{A}_{0}+i \eta$, $\mathscr{A}_{0}=(1 / 2 t|\eta|) \partial_{\eta}$, then we obtain the commutator $\partial_{\eta} \mathscr{V}=$ $2 t|\eta|[i \eta, \mathscr{V}]$. Also $\mathscr{V}=\mathscr{V}_{1}+\mathscr{V}_{2}$. Hence

$$
\begin{aligned}
\left\||\eta|^{1 / 2-j}\{\hat{\eta}\}^{v}\langle\hat{\eta}\rangle^{-v} \partial_{\eta} \mathscr{V} \xi^{j} \phi\right\|_{L^{2}} \\
\leq C t\left\||\eta|^{3 / 2-j}\{\widehat{\eta}\}^{v}\langle\hat{\eta}\rangle^{-v}\left[\eta, \mathscr{V}_{1}\right] \xi^{j} \phi\right\|_{\mathbf{L}^{2}} \\
+C t\left\||\eta|^{3 / 2-j}\{\hat{\eta}\}^{v}\langle\hat{\eta}\rangle^{-v} \mathscr{V}_{2} \xi^{j} \phi\right\|_{\mathbf{L}^{2}} \\
\quad+C t\left\||\eta|^{1 / 2-j}\{\hat{\eta}\}^{v}\langle\hat{\eta}\rangle^{-v} \mathscr{V}_{2} \xi^{j+1} \phi\right\|_{\mathbf{L}^{2}} .
\end{aligned}
$$

By Lemma 8 we find $\left\||\eta|^{3 / 2-j}\{\widehat{\eta}\}^{v}\langle\widehat{\eta}\rangle^{-v} t\left[\eta, \mathscr{V}_{1}\right] \xi^{j} \phi\right\|_{L^{2}} \leq$ $C t^{1 / 6}\|\phi\|_{\mathrm{Y}}$ for all $t \geq 1$, if $j \geq 0, v \in(0,1)$. Also by Lemma 9 we get $\left\||\eta|^{5 / 2-j}\{\widehat{\eta}\}^{v}\langle\widehat{\eta}\rangle^{-v} t \mathscr{V}_{2} \xi^{j} \phi\right\|_{L^{2}}+$ $\left\||\eta|^{3 / 2-j}\{\hat{\eta}\}^{\nu}\langle\hat{\eta}\rangle^{-v} t \mathscr{V}_{2} \xi^{j+1} \phi\right\|_{\mathrm{L}^{2}} \leq C t^{1 / 6}\|\phi\|_{\mathrm{Y}}$ for all $t \geq 1$, if $j=0,1, v \in(0,1)$. Hence the result of the lemma follows. Lemma 10 is proved.

\section{A Priori Estimates}

Local existence and uniqueness of solutions to Cauchy problem (1) were shown in $[19,20]$ when $u_{0} \in \mathbf{H}^{1}$. By using the local existence result, we have the following.

Theorem 11. Assume that the initial data $u_{0} \in \mathbf{H}^{1} \cap \mathbf{H}^{0,1}$. Then there exists a unique local solution $u$ of Cauchy problem (1) such that $\mathscr{U}(-t) u \in \mathbf{C}\left([0, T] ; \mathbf{H}^{1} \cap \mathbf{H}^{0,1}\right)$.

We can take $T>1$ if the data are small in $\mathbf{H}^{1} \cap \mathbf{H}^{0,1}$ and we may assume that $\|u\|_{\mathrm{X}_{1}} \leq \varepsilon$. To get the desired results, we prove a priori estimates of solutions uniformly in time. Define the following norm

$$
\begin{aligned}
& \|u\|_{\mathbf{X}_{T}}=\sup _{t \in[1, T]}\left(\|\widehat{\varphi}(t)\|_{\mathbf{L}^{\infty}}+t^{-1 / 6}\|\mathscr{J} u(t)\|_{\mathbf{L}^{2}}\right. \\
& \left.\quad+t^{(1 / 3)(1-1 / p)}\|u(t)\|_{\mathbf{L}^{p}}\right),
\end{aligned}
$$

where $\mathscr{J}=\mathscr{U}(t) x \mathscr{U}(-t), \widehat{\varphi}(t)=\mathscr{F} \mathscr{U}(-t) u(t)$, and $p>4$.

First we obtain the large time asymptotic behavior of the nonlinearity $\mathscr{F} \mathscr{U}(-t) \partial_{x}\left(|u|^{2} u\right)$.

Lemma 12. The asymptotics $t \mathscr{F} \mathscr{U}(-t) \partial_{x}\left(|u|^{2} u\right)=$ $i \widehat{\xi}|\bar{\xi}|^{5}\langle\widehat{\xi}\rangle^{-6}|\widehat{\varphi}|^{2} \widehat{\varphi}+O\left(\{\widehat{\xi}\}\langle\widehat{\xi}\rangle^{-\gamma}\|\widehat{\varphi}\|_{\mathrm{Y}}^{3}\right)$ is true for all $t \geq 1$ and $\xi \in \mathbb{R}$, where $\widehat{\varphi}(t)=\mathscr{F} \mathscr{U}(-t) u(t)$ and $v>0$ is small.

Proof. In view of factorization formula (11) we find $t \mathscr{F} \mathscr{U}(-t) \partial_{x}\left(|u|^{2} u\right)=i \xi \mathscr{V}^{*}(t)|\psi|^{2} \psi=\mathscr{V}^{*}(t) \mathscr{A}_{1}(t)|\psi|^{2} \psi$, where $\psi=\mathscr{V} \hat{\varphi}$. Then by Lemma 5 with $\alpha=1 / 2+\nu, \beta=2 v$, and $v>0$ small, we get

$$
\begin{aligned}
& t \mathscr{F} \mathcal{U}(-t) \partial_{x}\left(|u|^{2} u\right)=i \xi \mathscr{V}^{*}(t)|\psi|^{2} \psi=i \xi A^{*}|\psi|^{2} \psi \\
& +O\left(t ^ { - 1 / 2 + v / 3 } \hat { \xi } \left(\left\||\eta|^{1 / 2+v}\langle\hat{\eta}\rangle^{-2 v} \partial_{\eta}\left(|\psi|^{2} \psi\right)\right\|_{L^{2}}\right.\right. \\
& \left.\left.+\left\||\eta|^{-1 / 2+v}\langle\hat{\eta}\rangle^{-2 v}|\psi|^{2} \psi\right\|_{L^{2}}\right)\right)
\end{aligned}
$$

in the case of $|\xi|<t^{-1 / 3}$ and

$$
\begin{array}{rl}
t \mathscr{F} & \mathcal{U}(-t) \partial_{x}\left(|u|^{2} u\right)=\mathscr{V}^{*} \mathscr{A}_{1}|\psi|^{2} \psi=A^{*} \mathscr{A}_{1}|\psi|^{2} \psi \\
& +O\left(t^{\nu / 3-1 / 6}\langle\hat{\xi}\rangle^{-2 v}\right. \\
& \cdot\left(\left\||\eta|^{1 / 2+v}\langle\hat{\eta}\rangle^{-2 v} \partial_{\eta} \mathscr{A}_{1}\left(|\psi|^{2} \psi\right)\right\|_{L^{2}}\right. \\
& \left.\left.+\left\||\eta|^{-1 / 2+v}\langle\hat{\eta}\rangle^{-2 v} \mathscr{A}_{1}\left(|\psi|^{2} \psi\right)\right\|_{L^{2}}\right)\right)
\end{array}
$$

in the case of $|\xi|>t^{-1 / 3}$. Via identity $|\eta|^{\nu}=t^{-v / 3}\{\widehat{\eta}\}^{\nu}\langle\widehat{\eta}\rangle^{\nu}$, we consider the remainder terms

$$
\begin{aligned}
& \left\||\eta|^{1 / 2+\nu}\langle\widehat{\eta}\rangle^{-2 v} \partial_{\eta}\left(|\psi|^{2} \psi\right)\right\|_{L^{2}} \\
& \leq C\left\||\eta|^{1 / 2+v}\langle\widehat{\eta}\rangle^{-2 v}|\psi|^{2} \partial_{\eta} \psi\right\|_{\mathbf{L}^{2}} \\
& \leq C\left\||\eta|^{\nu}\{\widehat{\eta}\}^{-v}\langle\hat{\eta}\rangle^{-v}|\psi|^{2}\right\|_{L^{\infty}} \\
& \cdot\left\||\eta|^{1 / 2}\{\widehat{\eta}\}^{\nu}\langle\hat{\eta}\rangle^{-v} \partial_{\eta} \psi\right\|_{\mathbf{L}^{2}} \leq C t^{1 / 6-v / 3}\|\phi\|_{\mathbf{Y}} \\
& \cdot\left\||\psi|^{2}\right\|_{L^{\infty}}, \\
& \left\||\eta|^{-1 / 2+v}\langle\widehat{\eta}\rangle^{-2 v} \partial_{\eta} \mathscr{A}_{1}\left(|\psi|^{2} \psi\right)\right\|_{\mathrm{L}^{2}} \\
& \leq C\left\||\eta|^{1+\nu}\{\hat{\eta}\}^{-v}\langle\hat{\eta}\rangle^{-v}|\psi|^{2}\right\|_{\mathbf{L}^{\infty}} \\
& \cdot\left\||\eta|^{-1 / 2}\{\hat{\eta}\}^{\nu}\langle\hat{\eta}\rangle^{-v} \partial_{\eta} \psi_{1}\right\|_{\mathbf{L}^{2}} \\
& +C\left\||\eta|^{\nu}\{\hat{\eta}\}^{-v}\langle\hat{\eta}\rangle^{-v} \psi \psi_{1}\right\|_{L^{\infty}} \\
& \cdot\left\||\eta|^{1 / 2}\{\hat{\eta}\}^{\nu}\langle\hat{\eta}\rangle^{-v} \partial_{\eta} \psi\right\|_{L^{2}} \leq C t^{1 / 6-v / 3}\|\phi\|_{\mathbf{Y}} \\
& \cdot\left(\left\|\eta|\psi|^{2}\right\|_{\mathbf{L}^{\infty}}+\left\|\psi \psi_{1}\right\|_{\mathbf{L}^{\infty}}\right),
\end{aligned}
$$

where $\psi_{1}=\mathscr{V} i \xi \widehat{\varphi}$. By Lemma 10 with $j=0,1$, we have

$$
\begin{aligned}
& \left\||\eta|^{1 / 2}\{\hat{\eta}\}^{\nu}\langle\hat{\eta}\rangle^{-v} \partial_{\eta} \psi\right\|_{\mathbf{L}^{2}}+\left\||\eta|^{-1 / 2}\{\hat{\eta}\}^{\nu}\langle\hat{\eta}\rangle^{-v} \partial_{\eta} \psi_{1}\right\|_{\mathbf{L}^{2}} \\
& \quad \leq C t^{1 / 6}\|\hat{\varphi}\|_{\mathbf{Y}} .
\end{aligned}
$$

Using Lemma 13 we get $|\psi| \leq C\left(t^{1 / 2}|\eta|\langle\hat{\eta}\rangle^{-3 / 2}+\right.$ $\left.t^{1 / 6}\langle\widehat{\eta}\rangle^{-3 / 2}\right)\|\phi\|_{\mathrm{Y}} \leq C t^{1 / 6}\langle\widehat{\eta}\rangle^{-1 / 2}\|\widehat{\varphi}\|_{\mathrm{Y}}$ and $\left|\psi_{1}\right| \leq$ $C\left(t^{1 / 2}|\eta|^{2}\langle\widehat{\eta}\rangle^{-3 / 2}+t^{-1 / 6}\langle\hat{\eta}\rangle^{-1 / 2}\right)\|\phi\|_{\mathrm{Y}} \leq C\left(|\eta|^{1 / 2}+t^{-1 / 6}\right)\|\hat{\varphi}\|_{\mathrm{Y}}$. Hence $\left\|\psi^{2}\right\|_{L^{\infty}} \leq C t^{1 / 3}\|\hat{\varphi}\|_{Y}^{2}$ and $\left\|\eta|\psi|^{2}\right\|_{L^{\infty}}+\left\|\psi \psi_{1}\right\|_{L^{\infty}} \leq$ $C\|\widehat{\varphi}\|_{\mathrm{Y}}^{2}$. Also we find $\left\||\eta|^{-1 / 2+\nu}\langle\widehat{\eta}\rangle^{-2 v}|\psi|^{2} \psi\right\|_{L^{2}} \leq$ $C t^{1 / 2}\|\widehat{\varphi}\|_{\mathbf{Y}}^{3}\left\||\eta|^{-1 / 2+v}\langle\hat{\eta}\rangle^{-2 v-3 / 2}\right\|_{L^{2}} \leq C t^{1 / 2-v / 3}\|\widehat{\varphi}\|_{\mathbf{Y}}^{3}$ and $\left\||\eta|^{-1 / 2+\nu}\langle\widehat{\eta}\rangle^{-2 v} \mathscr{A}_{1}\left(|\psi|^{2} \psi\right)\right\|_{L^{2}} \leq C t^{1 / 6}\|\widehat{\varphi}\|_{\mathrm{Y}}^{3}\left\|\left.\eta\right|^{-1 / 2+\nu}\langle\widehat{\eta}\rangle^{-2 \nu-1 / 2}\right\|_{L^{2}} \leq$ $C t^{1 / 6-v / 3}\|\widehat{\varphi}\|_{\mathrm{Y}}^{3}$. Therefore we obtain $t \mathscr{F} \mathscr{U}(-t) \partial_{x}\left(|u|^{2} u\right)=$ $i \xi A^{*}|\psi|^{2} \psi+O\left(\{\widehat{\xi}\}\langle\widehat{\xi}\rangle^{-2 \gamma}\|\widehat{\varphi}\|_{\mathrm{Y}}^{3}\right)$ for $|\xi|<t^{-1 / 3}$ and $t \mathscr{F} \mathscr{U}(-t) \partial_{x}\left(|u|^{2} u\right)=A^{*} \mathscr{A}_{1}|\psi|^{2} \psi+O\left(\{\widehat{\xi}\}\langle\widehat{\xi}\rangle^{-2 v}\|\widehat{\varphi}\|_{\mathrm{Y}}^{3}\right)$ for $|\xi|>t^{-1 / 3}$. Next by Lemma 13 we have $\psi_{j}(t, \xi)=$ $\left(t^{1 / 2}|\xi|(i \xi)^{j} / \sqrt{2 i\left\langle t \xi^{3}\right\rangle}\right) \hat{\varphi}(\xi)+O\left(t^{1 / 6-j / 3}\langle\widehat{\xi}\rangle^{j-3 / 4}\|\widehat{\varphi}\|_{\mathrm{Y}}\right)$ for $j=0,1$. Then we get $i \xi A^{*}|\psi|^{2} \psi=i \widehat{\xi}|\xi|^{5}\langle\widehat{\xi}\rangle^{-6}|\widehat{\varphi}|^{2} \widehat{\varphi}+$ 
$O\left(\{\widehat{\xi}\}\langle\widehat{\xi}\rangle^{-\gamma}\|\widehat{\varphi}\|_{\mathrm{Y}}^{3}\right)$ and $A^{*} \mathscr{A}_{1}|\psi|^{2} \psi=i \widehat{\xi}|\widehat{\xi}|^{5}\langle\widehat{\xi}\rangle^{-6}|\widehat{\varphi}|^{2} \widehat{\varphi}+$ $O\left(\{\widehat{\xi}\}\langle\widehat{\xi}\rangle^{-v}\|\widehat{\varphi}\|_{\mathrm{Y}}^{3}\right)$. Lemma 12 is proved.

Next we estimate the solutions in the norm $\mathbf{X}_{T}$.

Lemma 13. Assume that $\|u\|_{\mathbf{X}_{1}} \leq \varepsilon$ holds. Then there exists $\varepsilon>0$ such that the estimate $\|u\|_{\mathbf{X}_{T}}<C \varepsilon$ is true for all $T>1$.

Proof. By continuity of the norm $\|u\|_{\mathbf{X}_{T}}$ with respect to $T$, arguing by the contradiction we can find the first time $T>0$ such that $\|u\|_{\mathbf{X}_{T}}=C \varepsilon$. To prove the estimate for the norm $\sup _{t \in[1, T]}\|\hat{\varphi}\|_{\mathbf{L}^{\infty}}<C \varepsilon$ we use (11). Then in view of Lemma 12, we get

$$
\begin{aligned}
\partial_{t} \widehat{\varphi} & =\lambda \mathscr{F} \mathcal{U}(-t) \partial_{x}\left(|u|^{2} u\right) \\
& =i \lambda t^{-1} \widehat{\xi}|\widehat{\xi}|^{5}\langle\widehat{\xi}\rangle^{-6}|\widehat{\varphi}|^{2} \widehat{\varphi}+O\left(\varepsilon^{3} t^{-1}\{\widehat{\xi}\}\langle\widehat{\xi}\rangle^{-\nu}\right) .
\end{aligned}
$$

For the case of $|\xi|<t^{-1 / 3}$ we can integrate $|\widehat{\varphi}(t, \xi)| \leq|\widehat{\varphi}(1, \xi)|+$ $C|\xi|\|\widehat{\varphi}\|_{\mathrm{Y}}^{3} \int_{1}^{t} \tau^{-2 / 3} d \tau \leq \varepsilon+C \xi t^{1 / 3} \varepsilon^{3} \leq \varepsilon+C \varepsilon^{3}$. For the case of $|\xi| \geq t^{-1 / 3}$ multiplying by $\overline{\widehat{\varphi}}$ and taking the real part of the result we obtain $\partial_{t}\left(|\widehat{\varphi}(t, \xi)|^{2}\right)=O\left(t^{-1}\{\widehat{\xi}\}\langle\widehat{\xi}\rangle^{-v}\|\widehat{\varphi}\|_{\mathrm{Y}}^{4}\right)$. Integrating in time we obtain

$$
\begin{aligned}
|\widehat{\varphi}(t, \xi)|^{2} \leq & \left|\widehat{\varphi}\left(\xi^{-3}, \xi\right)\right|^{2} \\
& +C\|\hat{\varphi}\|_{\mathbf{Y}}^{4} \int_{\xi^{-3}}^{t}\left\{\xi \tau^{1 / 3}\right\}\left\langle\xi \tau^{1 / 3}\right\rangle^{-v} \frac{d \tau}{\tau} \\
\leq & \varepsilon^{2}+C \varepsilon^{4} \int_{1}^{\xi t^{1 / 3}}\langle y\rangle^{-1-v} d y \leq \varepsilon^{2}+C \varepsilon^{4}
\end{aligned}
$$

Therefore $\|\mathscr{F} \mathscr{U}(-t) u(t)\|_{L^{\infty}}<C \varepsilon$. Applying estimate of Lemma 4 we find $\left|\partial_{x}^{j} u(t, x)\right| \leq C \varepsilon\left\langle x t^{-1 / 3}\right\rangle^{j / 2-1 / 4} t^{-1 / 3-j / 3}$, $\left|u(t, x) \partial_{x} u(t, x)\right| \leq C \varepsilon^{2} t^{-1}$, and

$$
\begin{aligned}
\|u\|_{\mathbf{L}^{p}} & \leq C t^{-1 / 3}\|u\|_{\mathbf{X}_{T}}\left\|\left\langle x t^{-1 / 3}\right\rangle^{-1 / 4}\right\|_{\mathbf{L}^{p}} \\
& \leq C \varepsilon t^{-(1 / 3)(1-1 / p)}
\end{aligned}
$$

if $p>4$. Consider a priori estimates for $\|\mathscr{J} u(t)\|_{\mathrm{L}^{2}}$. Using the identity $\partial_{x}^{-1} \mathscr{P} u-\mathscr{J} u=3 t \partial_{x}^{-1} \mathscr{L} u$, we get $\|\mathscr{J} u\|_{\mathrm{L}^{2}} \leq$ $C\left\|\partial_{x}^{-1} \mathscr{P} u\right\|_{\mathbf{L}^{2}}+C t\|u\|_{\mathbf{L}^{6}}^{3} \leq C\left\|\partial_{x}^{-1} \mathscr{P} u\right\|_{\mathbf{L}^{2}}+C \varepsilon^{3} t^{1 / 6}$. Applying the operator $\partial_{x}^{-1} \mathscr{P}$ to $(1)$, in view of the commutators $[\mathscr{L}, \mathscr{P}]=$ $3 \mathscr{L},\left[\mathscr{P}, \partial_{x}\right]=-\partial_{x}$, we get $\mathscr{L} \partial_{x}^{-1} \mathscr{P} u=\partial_{x}^{-1}(\mathscr{P}+3) \mathscr{L} u=$ $\lambda \partial_{x}^{-1}(\mathscr{P}+3) \partial_{x}\left(|u|^{2} u\right)=\lambda(\mathscr{P}+2)\left(|u|^{2} u\right)$. Then by the energy method we obtain

$$
\begin{aligned}
\frac{d}{d t}\left\|\partial_{x}^{-1} \mathscr{P} u\right\|_{\mathbf{L}^{2}}^{2} \leq & C\left\|u u_{x}\right\|_{\mathbf{L}^{\infty}}\left\|\partial_{x}^{-1} \mathscr{P} u\right\|_{\mathbf{L}^{2}}^{2} \\
& +\|u\|_{\mathbf{L}^{6}}^{3}\left\|\partial_{x}^{-1} \mathscr{P} u\right\|_{\mathbf{L}^{2}} \\
\leq & C \varepsilon^{2} t^{-1}\left\|\partial_{x}^{-1} \mathscr{P} u\right\|_{\mathbf{L}^{2}}^{2} \\
& +C \varepsilon^{3} t^{-5 / 6}\left\|\partial_{x}^{-1} \mathscr{P} u\right\|_{\mathbf{L}^{2}}
\end{aligned}
$$

from which it follows that $\left\|\partial_{x}^{-1} \mathscr{P} u\right\|_{L^{2}} \leq \varepsilon t^{C \varepsilon^{2}}+C \varepsilon^{3} t^{1 / 6}$. Therefore $\|\mathscr{J} u\|_{L^{2}} \leq C\left\|\partial_{x}^{-1} \mathscr{P} u\right\|_{L^{2}}+C t\|u\|_{L^{6}}^{3} \leq C \varepsilon^{3} t^{1 / 6}$ for all $t \in[1, T]$. Thus we obtain $\|u\|_{\mathbf{X}_{T}}<C \mathcal{E}$. Lemma 13 is proved.

\section{Proof of Theorem 1}

By Lemma 13 we see that a priori estimate $\|u\|_{\mathbf{X}_{T}} \leq C \varepsilon$ is true for all $T>0$. Therefore the global existence of solutions of Cauchy problem (1) satisfying the estimate $\|u\|_{\mathbf{X}_{\infty}} \leq$ $C \varepsilon$ follows by a standard continuation argument by local existence Theorem 11.

\section{Proof of Theorem 2}

In this section we prove the existence of a unique selfsimilar solution $v_{m}(t, x) \equiv t^{-1 / 3} f_{m}\left(x t^{-1 / 3}\right)$ for (1), which is uniquely determined by the total mass condition $m=$ $(1 / \sqrt{2 \pi}) \int_{\mathbf{R}} v_{m}(t, x) d x \neq 0$. Define the operators

$$
\begin{aligned}
\widetilde{\mathscr{V}} \phi & =\sqrt{\frac{1}{2 \pi}} \int_{\mathbb{R}} e^{-i S(\xi, \eta)} \phi(\xi) d \xi, \\
\widetilde{\mathscr{V}}^{*} \phi & =\sqrt{\frac{2}{\pi}} \int_{\mathbb{R}} e^{i S(\xi, \eta)} \phi(\eta)|\eta| d \eta .
\end{aligned}
$$

Then for the self-similar solutions $v_{m}(t, x)=$ $t^{-1 / 3} f_{m}\left(x t^{-1 / 3}\right)=\mathscr{D}_{t} \mathscr{B} M \mathscr{V} \widehat{\varphi_{m}}$, where $\widehat{\varphi_{m}}(t, \xi) \equiv$ $\mathscr{F} \mathscr{U}(-t) v_{m}(t)$, we find that $\widehat{\varphi_{m}}$ have a self-similar form, that is, $\widehat{\varphi_{m}}(t, \xi) \equiv \phi_{m}(\widehat{\xi})$ with $\widehat{\xi}=\xi t^{1 / 3}$. Using the relation $\partial_{t} \widehat{\varphi_{m}}(t, \xi)=(1 / 3) t^{-1} \eta \phi_{m}^{\prime}(\eta)$ by factorization formula (11) we get $(1 / 3) \eta \partial_{\eta} \phi_{m}(\eta)=i \lambda \eta \widetilde{\mathscr{V}}^{*}\left|\widetilde{\mathscr{V}} \phi_{m}\right|^{2} \widetilde{\mathscr{V}} \phi_{m}$. Therefore $\partial_{\eta} \phi_{m}(\eta)=3 i \lambda \widetilde{\mathscr{V}}^{*}\left|\widetilde{\mathscr{V}} \phi_{m}\right|^{2} \widetilde{\mathscr{V}} \phi_{m} \equiv F\left(\phi_{m}\right)$. Note that $F\left(\phi_{m}\right)$ is not in $\mathbf{L}^{2}$. Therefore we need the approximate equation. Define $\Theta(\eta)=1$ for $|\eta| \leq 1$ and $\Theta(\eta)=0$ for $|\eta|>2$, and denote $\Theta_{R}(\eta)=\Theta(\eta / R)$. Also define the approximate equation

$$
\begin{aligned}
\partial_{\eta} \phi_{m, R}(\eta) & =3 i \lambda \widetilde{\mathscr{V}}^{*} \Theta_{R}\left|\widetilde{\mathscr{V}} \Theta_{R} \phi_{m, R}\right|^{2} \widetilde{\mathscr{V}} \Theta_{R} \phi_{m, R} \\
& \equiv F_{R}\left(\phi_{m, R}(\eta)\right) .
\end{aligned}
$$

Let us show a priori estimate $\left\|\phi_{m, R}\right\|_{\mathbf{Z}}=\left\|\phi_{m, R}\right\|_{L^{\infty}}+$ $\left\|\partial_{\eta} \phi_{m, R}\right\|_{\mathrm{L}^{2}} \leq 3|m|$ uniformly in $R$. Applying Lemma 12 with $t=1$ we get

$$
\begin{aligned}
\partial_{\eta} \phi_{m, R}(\eta)= & 3 i \lambda|\eta|^{5}\langle\eta\rangle^{-6}\left|\Theta_{R} \phi_{m, R}(\eta)\right|^{2} \Theta_{R} \phi_{m, R}(\eta) \\
& +O\left(\langle\eta\rangle^{-1-v}\left\|\phi_{m, R}\right\|_{\mathrm{Z}}^{3}\right)
\end{aligned}
$$

Integrating with respect to $\eta$, we obtain $\left\|\partial_{\eta} \phi_{m, R}\right\|_{L^{2}} \leq$ $C\left\|\phi_{m, R}\right\|_{\mathrm{Z}}^{3}$. Also multiplying by $\overline{\phi_{m, R}}$ and integrating with respect to $\eta$ we get $\left|\phi_{m, R}(\eta)\right|=m+O\left(\int_{0}^{\eta}\langle\eta\rangle^{-1-\gamma}\left\|\phi_{m, R}\right\|_{\mathbf{Z}}^{3} d \eta\right)$. Hence $\left\|\phi_{m, R}\right\|_{\mathbf{L}^{\infty}} \leq|m|+C\left\|\phi_{m, R}\right\|_{\mathbf{Z}}^{3}$. Thus we obtain $\left\|\phi_{m, R}\right\|_{\mathbf{Z}} \leq$ $2|m|+C\left\|\phi_{m, R}\right\|_{Z}^{3} \leq 3|m|$ for some small $m$. Taking the limit $R \rightarrow \infty$, we find that there exists a unique solution 
$\phi_{m}$ of equation $\partial_{\eta} \phi_{m}(\eta)=3 i \lambda \widetilde{\mathscr{V}}^{*}\left|\widetilde{\mathscr{V}} \phi_{m}\right|^{2} \widetilde{\mathscr{V}} \phi_{m}$ in $\mathbf{Z}$. By the definition of $\phi_{m}(\eta)$, we obtain $\left\|\partial_{\xi} \widehat{\varphi_{m}}\right\|_{\mathbf{L}^{2}}=t^{1 / 6}\left\|\partial_{\eta} \phi_{m}\right\|_{\mathbf{L}^{2}} \leq$ $C|m| t^{1 / 6}$ and $\left\|\widehat{\varphi_{m}}\right\|_{\mathbf{L}^{\infty}} \leq 3|m|$. In the same way as in the proof of (73) we have $\mathbf{L}^{p}$ estimate of $v_{m}$ for $p>4$.

\section{Proof of Theorem 3}

Define the norm $\|u\|_{\mathbf{Y}_{T}}=\sup _{t \in[1, T]}\left(t^{1 / 2-\gamma}\|u\|_{\mathbf{L}^{\infty}}+t^{-\gamma}\|\mathscr{J} u\|_{\mathbf{L}^{2}}\right)$ with a small $\gamma>0$.

Lemma 14. Suppose that $\left\|u_{j}\right\|_{\mathbf{X}_{T}} \leq C \varepsilon, j=1,2$, where $\varepsilon$ is sufficiently small. Let $\widehat{\varphi_{1}}(t, 0)=\widehat{\varphi_{2}}(t, 0)$ for $j=1,2, t \geq 1$, where $\widehat{\varphi_{j}}(t, \xi)=\mathscr{F} \mathscr{U}(-t) u_{j}(t)$. Let $u_{2}=t^{-1 / 3} f\left(x t^{-1 / 3}\right)$ be a self-similar solution. Then the estimate $\left\|u_{1}-u_{2}\right\|_{\mathbf{Y}_{T}}<C \varepsilon$ is true for all $T>1$.

Proof. By the continuity of the norm $\left\|u_{1}-u_{2}\right\|_{\mathbf{Y}_{T}}$ with respect to $T$, arguing by the contradiction we can find for the first time $T>0$ such that $\left\|u_{1}-u_{2}\right\|_{\mathbf{Y}_{T}}=C \varepsilon$. We denote $\widehat{w}=\widehat{\varphi_{1}}-\widehat{\varphi_{2}}$ and $y=u_{1}-u_{2}$. Applying estimate of Lemma 4 we find

$$
\begin{aligned}
|y(t, x)| \leq & C t^{-1 / 3}\left\langle x^{1 / 2} t^{-1 / 6}\right\rangle^{-1 / 2}\left|\widehat{w}\left(t, x^{1 / 2} t^{-1 / 2}\right)\right| \\
& +C t^{-1 / 2}\left\langle x^{1 / 2} t^{-1 / 6}\right\rangle^{-3 / 4}\left\|\partial_{\xi} \widehat{w}\right\|_{L^{2}} \\
\leq & C t^{-1 / 2}\left\|\partial_{\xi} \widehat{w}\right\|_{L^{2}} .
\end{aligned}
$$

Thus we need to estimate the norm $\left\|\partial_{\xi} \widehat{w}\right\|_{\mathbf{L}^{2}}=\|\mathscr{F} y\|_{\mathbf{L}^{2}}$. For the difference $y$ we get from (1) $\mathscr{L} \partial_{x}^{-1} \mathscr{P} y=\lambda(\mathscr{P}+2)\left(\left|u_{1}\right|^{2} u_{1}\right.$ $\left.\left|u_{2}\right|^{2} u_{2}\right)$. Hence by the energy method

$$
\begin{aligned}
\frac{d}{d t} \| & \partial_{x}^{-1} \mathscr{P} y \|_{\mathbf{L}^{2}}^{2} \\
= & 4 \operatorname{Re} \int_{\mathbf{R}} \overline{\partial_{x}^{-1} \mathscr{P} y}\left(\left|u_{1}\right|^{2} \mathscr{P} u_{1}-\left|u_{2}\right|^{2} \mathscr{P} u_{2}\right) d x \\
& +2 \operatorname{Re} \int_{\mathbf{R}} \overline{\partial_{x}^{-1} \mathscr{P} y}\left(u_{1}^{2} \overline{\mathscr{P} u_{1}}-u_{2}^{2} \overline{\mathscr{P} u_{2}}\right) d x \\
& +4 \operatorname{Re} \int_{\mathbf{R}} \overline{\partial_{x}^{-1} \mathscr{P} y}\left(\left|u_{1}\right|^{2} u_{1}-\left|u_{2}\right|^{2} u_{2}\right) d x .
\end{aligned}
$$

Next we get

$$
\begin{aligned}
4 \operatorname{Re} \int_{\mathbf{R}} \overline{\partial_{x}^{-1} \mathscr{P} y}\left(\left|u_{1}\right|^{2} \mathscr{P} u_{1}-\left|u_{2}\right|^{2} \mathscr{P} u_{2}\right) d x \\
=4 \operatorname{Re} \int_{\mathbf{R}}\left|u_{1}\right|^{2} \overline{\partial_{x}^{-1} \mathscr{P} y} \mathscr{P} y d x \\
\quad+4 \operatorname{Re} \int_{\mathbf{R}}\left(\left|u_{1}\right|^{2}-\left|u_{2}\right|^{2}\right) \mathscr{P} u_{2} \partial_{x}^{-1} \mathscr{P} y d x \\
=4 \operatorname{Re} \int_{\mathbf{R}} \partial_{x}\left|u_{1}\right|^{2}\left|\partial_{x}^{-1} \mathscr{P} y\right|^{2} d x \\
\quad+4 \operatorname{Re} \int_{\mathbf{R}}\left(\left|u_{1}\right|^{2}-\left|u_{2}\right|^{2}\right) \mathscr{P} u_{2} \overline{\partial_{x}^{-1} \mathscr{P} y} d x
\end{aligned}
$$

$$
\begin{aligned}
\leq & C\left\|\left|u_{1}\right| u_{1 x}\right\|_{L^{\infty}}\left\|\partial_{x}^{-1} \mathscr{P} y\right\|_{L^{2}}^{2} \\
& +C\left\|\partial_{x}^{-1} \mathscr{P} y\right\|_{L^{2}}\left\|\left(\left|u_{1}\right|^{2}-\left|u_{2}\right|^{2}\right) \mathscr{P} u_{2}\right\|_{L^{2}} .
\end{aligned}
$$

In the same manner

$$
\begin{aligned}
2 \operatorname{Re} \int_{\mathbf{R}} & \overline{\partial_{x}^{-1} \mathscr{P} y}\left(u_{1}^{2} \overline{\mathscr{P} u_{1}}-u_{2}^{2} \overline{\mathscr{P} u_{2}}\right) d x \\
= & 2 \operatorname{Re} \int_{\mathbf{R}} u_{1}^{2} \overline{\partial_{x}^{-1} \mathscr{P} y} \overline{\mathscr{P} y} d x \\
& +2 \operatorname{Re} \int_{\mathbf{R}}\left(u_{1}^{2}-u_{2}^{2}\right) \overline{\mathscr{P} u_{2}} \overline{\partial_{x}^{-1} \mathscr{P} y} d x \\
= & 2 \operatorname{Re} \int_{\mathbf{R}} \partial_{x} u_{1}^{2} \overline{\partial_{x}^{-1} \mathscr{P} y} d x \\
& +2 \operatorname{Re} \int_{\mathbf{R}}\left(u_{1}^{2}-u_{2}^{2}\right) \overline{\mathscr{P} u_{2}} \overline{\partial_{x}^{-1} \mathscr{P} y} d x \\
\leq & C\left\|u_{1} u_{1 x}\right\|_{\mathbf{L}^{\infty}}\left\|\partial_{x}^{-1} \mathscr{P} y\right\|_{\mathbf{L}^{2}}^{2} \\
& +C\left\|\partial_{x}^{-1} \mathscr{P} y\right\|_{\mathbf{L}^{2}}\left\|\left(u_{1}^{2}-u_{2}^{2}\right) \mathscr{P} u_{2}\right\|_{\mathbf{L}^{2}} .
\end{aligned}
$$

Note that $\mathscr{P} u_{2}=\partial_{x} x t^{-1 / 3} f\left(x t^{-1 / 3}\right)+3 t \partial_{t} t^{-1 / 3} f\left(x t^{-1 / 3}\right)=0$ for the case of self-similar solution $u_{2}=t^{-1 / 3} f\left(x t^{-1 / 3}\right)$. Hence

$$
\begin{aligned}
& \frac{d}{d t}\left\|\partial_{x}^{-1} \mathscr{P} y\right\|_{L^{2}}^{2} \\
& \leq C\left\|\left|u_{1}\right| u_{1 x}\right\|_{L^{\infty}}\left\|\partial_{x}^{-1} \mathscr{P} y\right\|_{L^{2}}^{2} \\
& \quad+C\left\|\partial_{x}^{-1} \mathscr{P} y\right\|_{L^{2}}\left\|\left|u_{1}\right|^{2} u_{1}-\left|u_{2}\right|^{2} u_{2}\right\|_{L^{2}} .
\end{aligned}
$$

By (78) we have $\left\langle x^{1 / 2} t^{-1 / 6}\right\rangle^{1 / 2}|u(t, x)| \leq C \varepsilon t^{-1 / 3}$. To estimate $\left\|\left|u_{1}\right|^{2} u_{1}-\left|u_{2}\right|^{2} u_{2}\right\|_{L^{2}}$ we use the above estimates to get

$$
\begin{aligned}
& \left\|\left|u_{1}\right|^{2} u_{1}-\left|u_{2}\right|^{2} u_{2}\right\|_{L^{2}} \\
& \leq C \sum_{j=1}^{2}\left\|\left\langle|x|^{1 / 2} t^{-1 / 6}\right\rangle^{1 / 2} u_{j}\right\|_{L^{\infty}}^{2}\left\|\left\langle|x|^{1 / 2} t^{-1 / 6}\right\rangle^{-1} y\right\|_{L^{2}} \\
& \leq C \varepsilon^{2} t^{-2 / 3}\left\|\left\langle|x|^{1 / 2} t^{-1 / 6}\right\rangle^{-1} y\right\|_{L^{2}} .
\end{aligned}
$$

In view of Lemma 4

$$
\begin{aligned}
& \left\|\left\langle|x|^{1 / 2} t^{-1 / 6}\right\rangle^{-1} y\right\|_{L^{2}} \\
& \leq C t^{-1 / 3}\left\|\left\langle|x|^{1 / 2} t^{-1 / 6}\right\rangle^{-3 / 2} \widehat{w}\left(t, x^{1 / 2} t^{-1 / 2}\right)\right\|_{\mathbf{L}_{x}^{2}} \\
& \quad+C t^{-1 / 2}\left\|\left\langle|x|^{1 / 2} t^{-1 / 6}\right\rangle^{-7 / 4}\right\|_{L^{2}}\left\|\partial_{\xi} \widehat{w}\right\|_{L^{2}} .
\end{aligned}
$$

Since $\widehat{w}(0)=0$, we get by the Hardy inequality $\left\|\left\langle|x|^{1 / 2} t^{-1 / 6}\right\rangle^{-3 / 2} \widehat{w}\left(t, x^{1 / 2} t^{-1 / 2}\right)\right\|_{\mathbf{L}_{x}^{2}} \leq C\left\|\partial_{\xi} \widehat{w}\right\|_{\mathbf{L}^{2}}$ and by a direct calculation $\left\|\left\langle|x|^{1 / 2} t^{-1 / 6}\right\rangle^{-7 / 4}\right\|_{L^{2}} \leq C t^{1 / 6}$. Hence 
$\left\|\left\langle|x|^{1 / 2} t^{-1 / 6}\right\rangle^{-1} y\right\|_{L^{2}} \leq C t^{-1 / 3}\left\|\partial_{\xi} \widehat{w}\right\|_{L^{2}}$. Therefore by (83) $\left\|\left|u_{1}\right|^{2} u_{1}-\left|u_{2}\right|^{2} u_{2}\right\|_{L^{2}} \leq C \varepsilon^{2} t^{-1}\left\|\partial_{\xi} \widehat{w}\right\|_{L^{2}} \leq C \varepsilon^{3} t^{-1+\gamma}$. Thus we obtain from (82) $(d / d t)\left\|\partial_{x}^{-1} \mathscr{P} y\right\|_{L^{2}} \leq C \varepsilon^{3} t^{-1+\gamma}$ which implies $\left\|\partial_{x}^{-1} \mathscr{P} y\right\|_{L^{2}} \leq C \varepsilon^{3} t^{\gamma}$. Therefore $\|\mathscr{J} y\|_{\mathbf{L}^{2}} \leq\left\|\partial_{x}^{-1} \mathscr{P} y\right\|_{\mathbf{L}^{2}}+$ $C t\left\|\left|u_{1}\right|^{2} u_{1}-\left|u_{2}\right|^{2} u_{2}\right\|_{L^{2}} \leq C \varepsilon^{3} t^{\gamma}$. Lemma 14 is proved.

Now we turn to the proof of asymptotic formula (7) for the solutions $u$ of Cauchy problem (1). Let $v_{m}(t, x)$ be the self-similar solution with the total mass condition $m=$ $(1 / \sqrt{2 \pi}) \int_{\mathbf{R}} u_{0}(x) d x=(1 / \sqrt{2 \pi}) \int_{\mathbf{R}} v_{m}(t, x) d x \neq 0$. Note that $\left\|v_{m}\right\|_{\mathbf{X}_{\infty}} \leq C \varepsilon$ by Theorem 2 and $\|u\|_{\mathbf{X}_{\infty}} \leq C \varepsilon$ by Theorem 1 . Also $m=\widehat{\varphi}(t, 0)=\widehat{v_{m}}(t, 0)$ for $t \geq 1$. Then by Lemma 14 we find $u(t, x)=v_{m}(t, x)+O\left(\varepsilon t^{-1 / 2+\gamma}\right)$. Thus asymptotics (7) follows. Theorem 3 is proved.

\section{Data Availability}

No data were used to support this study.

\section{Conflicts of Interest}

The authors declare that there are no conflicts of interest regarding the publication of this paper.

\section{Acknowledgments}

The work is partially supported by CONACYT 252053-F and PAPIIT Project IN100817.

\section{References}

[1] F. J. Diaz-Otero and P. Chamorro-Posada, "Interchannel soliton collisions in periodic dispersion maps in the presence of third order dispersion," Journal of Nonlinear Mathematical Physics, vol. 15, pp. 137-143, 2008.

[2] J. R. Taylor, Generation and compression of femtosecond solitons in optical fibers, in Optical Solitons-Theory and Experiment, J. R. Taylor, Ed., Cambridge Studies in Modern Optics, Cambridge University Press, Cambridge, UK, 1992.

[3] S. Cui and S. Tao, "Strichartz estimates for dispersive equations and solvability of the Kawahara equation," Journal of Mathematical Analysis and Applications, vol. 304, no. 2, pp. 683-702, 2005.

[4] S. B. Cui, D. G. Deng, and S. P. Tao, "Global existence of solutions for the Cauchy problem of the Kawahara equation with L2 initial data," Acta Mathematica Sinica, vol. 22, no. 5, pp. 1457-1466, 2006.

[5] Y. Kodama and A. Hasegawa, "Nonlinear pulse propagation in a monomode dielectric guide," IEEE Journal of Quantum Electronics, vol. 23, no. 5, pp. 510-524, 1987.

[6] A. T. Il'ichev and A. Y. Semenov, "Stability of solitary waves in dispersive media described by a fifth-order evolution equation," Theoretical and Computational Fluid Dynamics, vol. 3, no. 6, pp. 307-326, 1992.

[7] T. Kawahara, "Oscillatory solitary waves in dispersive media," Journal of the Physical Society of Japan, vol. 33, no. 1, pp. 260264, 1972.

[8] Y. Kodama, "Optical solitons in a monomode fiber," Journal of Statistical Physics, vol. 39, no. 5-6, pp. 597-614, 1985.
[9] A. Mussot, A. Kudlinski, E. Louvergneaux, M. Kolobov, and M. Taki, "Impact of the third-order dispersion on the modulation instability gain of pulsed signals," Optics Expresss, vol. 35, no. 8, pp. 1194-1196, 2010.

[10] I. Naumkin and R. Weder, "High-energy and smoothness asymptotic expansion of the scattering amplitude for the Dirac equation and application," Mathematical Methods in the Applied Sciences, vol. 38, no. 12, pp. 2427-2465, 2015.

[11] M. Taki, A. Mussot, A. Kudlinski, E. Louvergneaux, M. Kolobov, and M. Douay, "Third-order dispersion for generating optical rogue solitons," Physics Letters A, vol. 374, no. 4, pp. 691-695, 2010.

[12] X. Carvajal, "Local well-posedness for a higher order nonlinear Schrödinger equation in Sobolev spaces of negative indices," Electronic Journal of Differential Equations, vol. 13, pp. 1-13, 2004.

[13] X. Carvajal and F. Linares, "A higher order nonlinear Schrödinger equation with variable coefficients," Differential Integral Equations, vol. 16, no. 9, pp. 1111-1130, 2003.

[14] C. Laurey, "The Cauchy problem for a third order nonlinear Schrödinger equation," Nonlinear Analysis: Theory, Methods \& Applications, vol. 29, no. 2, pp. 121-158, 1997.

[15] J. L. Bona, G. Ponce, J.-C. Saut, and C. Sparber, "Dispersive blow-up for nonlinear Schrödinger equations revisited," Journal de Mathématiques Pures et Appliquées, vol. 102, no. 4, pp. 782811, 2014.

[16] J. Ginibre, Y. Tsutsumi, and G. Velo, "Existence and uniqueness of solutions for the generalized Korteweg de Vries equation," Mathematische Zeitschrift, vol. 203, no. 1, pp. 9-36, 1990.

[17] N. Hayashi, "Analyticity of solutions of the Korteweg-de Vries equation," SIAM Journal on Mathematical Analysis, vol. 22, no. 6, pp. 1738-1743, 1991.

[18] T. Kato, "On the Cauchy problem for the (generalized) Korteweg-de Vries equation," in Studies in Applied Mathematics, vol. 8 of Adv. Math. Suppl. Stud., pp. 93-128, Academic Press, New York, 1983.

[19] C. E. Kenig, G. Ponce, and L. Vega, "On the (generalized) Korteweg-de Vries equation," Duke Mathematical Journal, vol. 59, no. 3, pp. 585-610, 1989.

[20] C. E. Kenig, G. Ponce, and L. Vega, "Well-posedness of the initial value problem for the Korteweg-de Vries equation," Journal of the American Mathematical Society, vol. 4, no. 2, pp. 323-347, 1991.

[21] C. E. Kenig, G. Ponce, and L. Vega, "Well-posedness and scattering results for the generalized Korteweg-de Vries equation via the contraction principle," Communications on Pure and Applied Mathematics, vol. 46, no. 4, pp. 527-620, 1993.

[22] S. N. Kruzhkov, A. V. Faminskiŭ, and J. R. Schulenberger, "Generalized Solutions of the Cauchy Problem for the Korteweg-de Vries Equation," Mathematics of the USSR - Sbornik, vol. 48, no. 2, pp. 391-421, 1984.

[23] J.-C. Saut, "Sur quelques généralizations de l'équation de Korteweg- de Vries," Journal de Mathématiques Pures et Appliquées, vol. 58, no. 1, pp. 21-61, 1979.

[24] G. Staffilani, "On the generalized Korteweg-de Vries-type equations," Differential Integral Equations, vol. 10, no. 4, pp. 777-796, 1997.

[25] M. Tsutsumi, "On global solutions of the generalized Kortwegde Vries equation," Publications of the Research Institute for Mathematical Sciences, vol. 7, pp. 329-344, 1971/72. 
[26] A. De Bouard, N. Hayashi, and K. Kato, "Gevrey regularizing effect for the (generalized) Korteweg - de Vries equation and nonlinear Schrödinger equations," Annales de l'Institut Henri Poincaré C, Analyse Non Linéaire, vol. 12, no. 6, pp. 673-725, 1995.

[27] T. Cazenave and I. Naumkin, "Modified scattering for the critical nonlinear Schrödinger equation," Journal of Functional Analysis, vol. 274, no. 2, pp. 402-432, 2018.

[28] P. Constantin and J.-C. Saut, "Local smoothing properties of dispersive equations," Journal of the American Mathematical Society, vol. 1, no. 2, pp. 413-439, 1988.

[29] W. Craig, K. Kappeler, and W. A. Strauss, "Gain of regularity for solutions of KdV type," Annales de l'Institut Henri Poincaré C, Analyse Non Linéaire, vol. 9, no. 2, pp. 147-186, 1992.

[30] S. Klainerman, "Long-time behavior of solutions to nonlinear evolution equations," Archive for Rational Mechanics and Analysis, vol. 78, no. 1, pp. 73-98, 1982.

[31] S. Klainerman and G. Ponce, "Global, small amplitude solutions to nonlinear evolution equations," Communications on Pure and Applied Mathematics, vol. 36, no. 1, pp. 133-141, 1983.

[32] J. L. Bona and J.-C. Saut, "Dispersive blowup of solutions of generalized Korteweg-de Vries equations," Journal of Differential Equations, vol. 103, no. 1, pp. 3-57, 1993.

[33] N. Hayashi and E. I. Kaikina, "Asymptotics for the thirdorder nonlinear Schrödinger equation in the critical case," Mathematical Methods in the Applied Sciences, vol. 40, no. 5, pp. 1573-1597, 2017.

[34] N. Hayashi, J. A. Mendez-Navarro, and P. Naumkin, "Asymptotics for the fourth-order nonlinear Schrödinger equation in the critical case," Journal of Differential Equations, vol. 261, no. 9, pp. 5144-5179, 2016.

[35] N. Hayashi and P. I. Naumkin, "The initial value problem for the cubic nonlinear Klein-Gordon equation," Zeitschrift fur Angewandte Mathematik und Physik, vol. 59, no. 6, pp. 10021028, 2008.

[36] N. Hayashi and T. Ozawa, "Scattering theory in the weighted L2(Rn) spaces for some Schr ödinger equations," Annales de l'Institut Henri Poincaré. Section A, Physique Théorique, vol. 48, no. 1, pp. 17-37, 1988.

[37] I. P. Naumkin, "Sharp asymptotic behavior of solutions for cubic nonlinear Schrödinger equations with a potential," Journal of Mathematical Physics, vol. 57, no. 5, Article ID 051501, 051501, 31 pages, 2016.

[38] I. P. Naumkin, "Initial-boundary value problem for the one dimensional Thirring model," Journal of Differential Equations, vol. 261, no. 8, pp. 4486-4523, 2016.

[39] M. V. Fedoryuk, "Asymptotics: integrals and series," in Mathematical Reference Library, Nauka, vol. 13, p. 544, SpringerVerlag, Moscow, 1987.

[40] E. M. Stein and R. Shakarchi, "Functional analysis," in Introduction to Further Topics in Analysis, vol. 4 of Princeton Lectures in Analysis, Princeton University Press, Princeton, NJ, USA, 2011. 


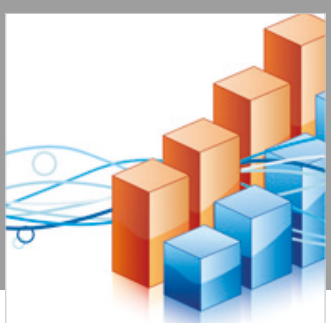

Advances in

Operations Research

\section{-n-m}
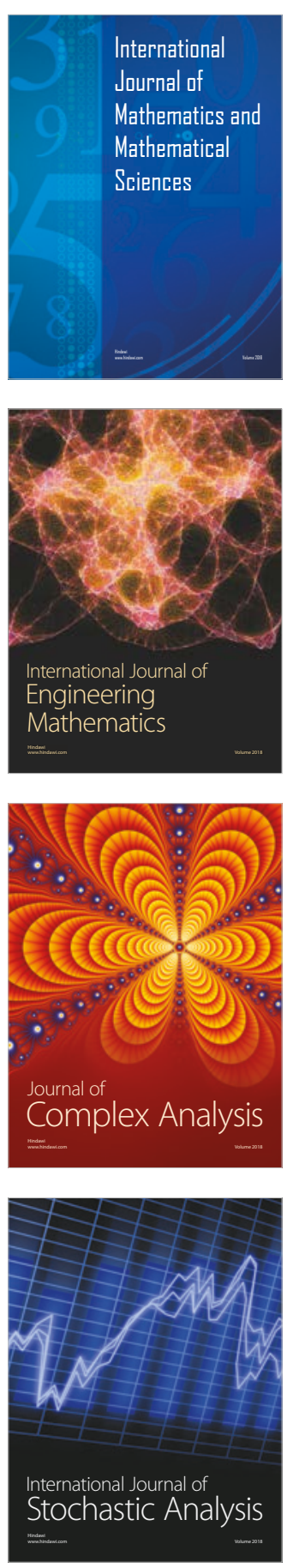
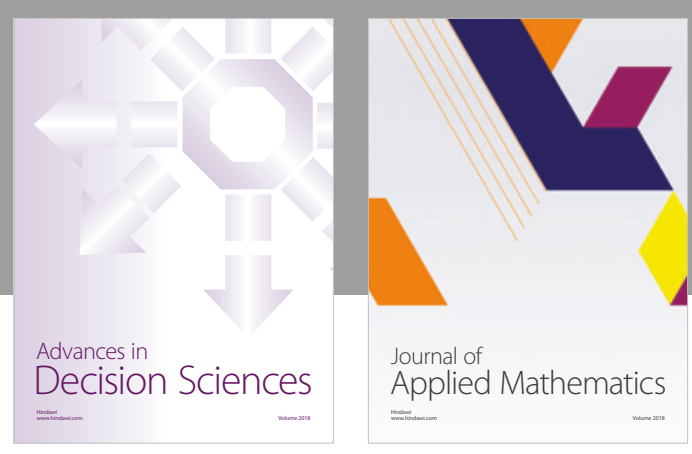

Journal of

Applied Mathematics
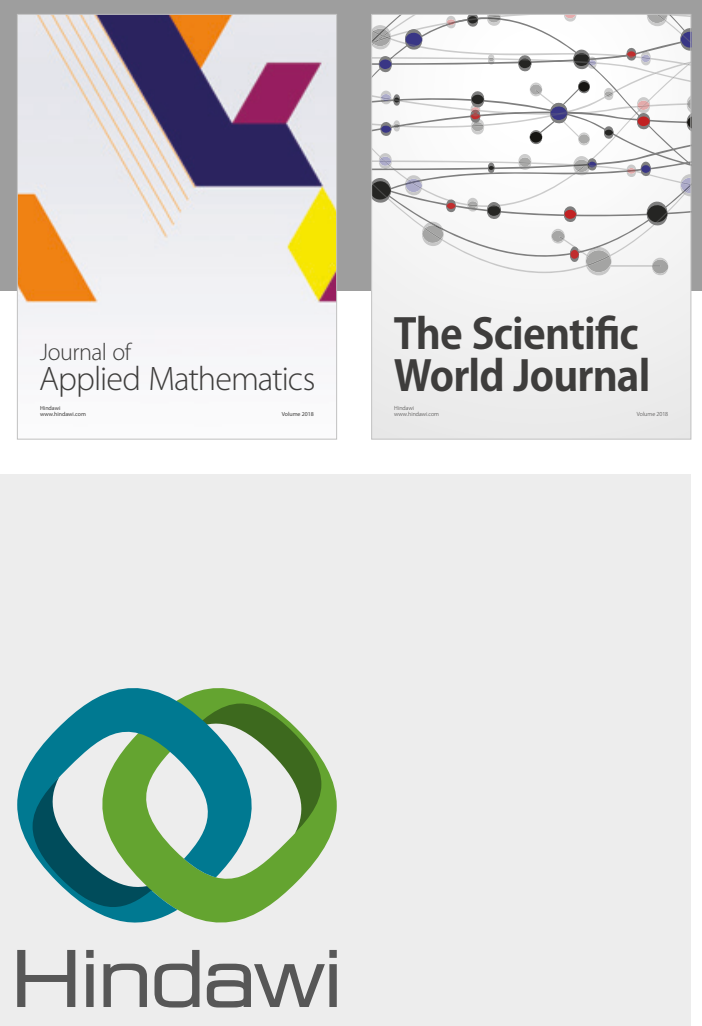

Submit your manuscripts at

www.hindawi.com

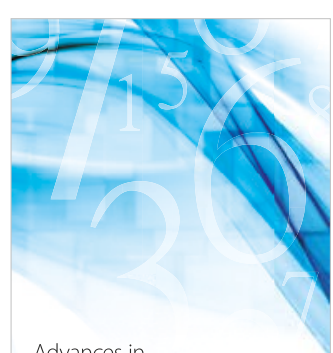

Advances in
Numerical Analysis
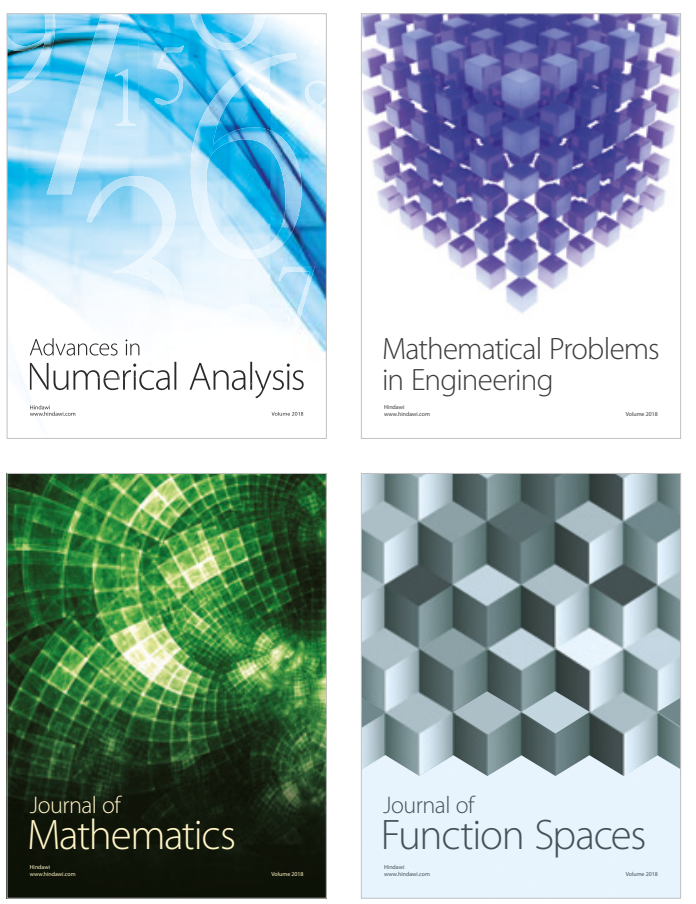

Mathematical Problems in Engineering

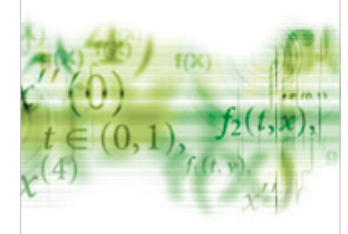

International Journal of

Differential Equations

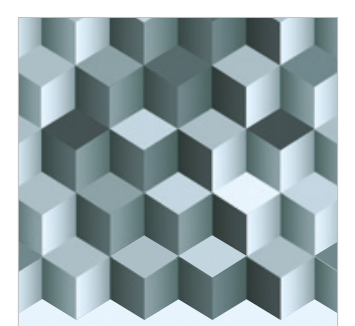

Journal of

Function Spaces

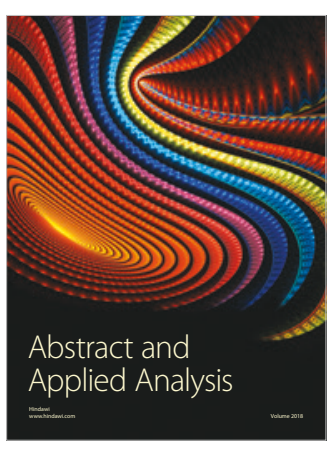

The Scientific

World Journal

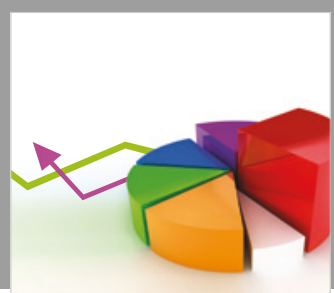

Journal of

Probability and Statistics
